\title{
On the Geometry of Lorentz Orbit Spaces
}

\author{
A. Gheorghe` and El. Mihul` \\ Joint Institute for Nuclear Research, Dubna, USSR \\ Received March 12, 1974; in revised form August 13, 1974
}

\begin{abstract}
The orbit space of the Lorentz group acting on the product of $n$ real, or complex, Minkowski spaces is stratified into subspaces isomorphic to certain products of Grassmann manifolds and varieties of Gram matrices. The Lorentz orbits (of nonzero dimension) are completely classified by the Stiefel manifolds of standard orthogonal bases for the linear subspaces of the Minkowski space. Several representations of the spaces of $n$-point Lorentz invariant distributions and differentiable, or analytic, functions onto appropriate spaces of distributions and functions of Lorentz invariant variables are also discussed.
\end{abstract}

\section{Introduction}

Considerable effort has been expended in many physical schemes for the study of Lorentz invariant functions and distributions. Excepting the case of the Lorentz invariant polynomials [1], the following problem is not completely solved: find a space of distributions or functions of Lorentz invariant variables isomorphic to a given space of $n$-point Lorentz invariant distributions or differentiable (resp. analytic) Lorentz invariant functions defined on a differentiable (resp. analytic) Lorentz invariant submanifold of the product of $n$ real (resp. complex) Minkowski spaces. This problem has been extensively studied by Hall, Wightman, and Bargmann [2], Hepp [3], Stapp, Minkowski, Williams, and Seiler [4] for Lorentz invariant functions on saturated domains and by Methée [5] for Lorentz invariant one-point distributions. Here a saturated domain is a domain which contains the closures of all Lorentz orbits of its points. The origin of the difficulty in solving the abovementioned problem is that the product of $n$ Minkowski spaces contains non-saturated domains and the corresponding Lorentz orbit space is not a Hausdorff one. In order to exhibit this difficulty in concrete terms, the purpose of this work is to give a minimal decomposition of the Lorentz orbit space corresponding to the product of $n$ Minkowski spaces into Lorentz orbit subspaces homeomorphic to certain products of manifolds of Gram matrices and Grassmann manifolds. Some algebraic and analytic properties of this decomposition and a complete

\footnotetext{
* On leave of absence from the Institute of Atomic Physics, Bucharest, Romania.
} 
classification of the considered Lorentz orbits using the Stiefel manifolds of standard orthogonal bases for the linear subspaces of the Minkowski space are also presented in the following.

\section{Stiefel Manifolds for the Minkowski Space}

In this section, we describe the geometric structure of certain families of orthogonal bases for the Minkowski space and its linear subspaces by analogy with the Stiefel manifolds used in the orthogonal geometry of index zero [6]. It is convenient to review briefly some definitions, notation, and the elementary technique of orthogonal geometry applied to the Minkowski space (several details can be found in Refs. $[2,4]$ and [7]).

Throughout this paper, $\boldsymbol{k}$ denotes either the field $\boldsymbol{R}$ of real numbers or the field $\boldsymbol{C}$ of complex numbers. However, the subsequent algebraic results are satisfied for certain large classes of fields which will be specified later.

The Minkowski space $M_{m}(\boldsymbol{k})$ is the $m$-dimensional linear space over $\boldsymbol{k}$ of all $m$-ples $x=\left(x^{0}, x^{1}, \ldots, x^{m-1}\right)$ of numbers belonging to $\boldsymbol{k}$, endowed with the Euclidean topology and the Minkowski scalar product [i.e. the symmetric bilinear $\boldsymbol{k}$-form defined by $(x, y) \rightarrow x y$ $=x^{0} y^{0}-x^{1} y^{1}-\cdots-x^{m-1} y^{m-1}$ for any $\left.x, y \in M_{m}(\boldsymbol{k})\right]$.

Let $F_{m}(\boldsymbol{k})$ denote the set of all linear subspaces of $M_{m}(\boldsymbol{k})$. The radical $\operatorname{rad} N$ of $N \in F_{m}(\boldsymbol{k})$ is the linear subspace of $N$ consisting of all $x \in N$ which satisfy the relation $x y=0$ for any $y \in N$. The index $s_{m}(\boldsymbol{k})$ of $M_{m}(\boldsymbol{k})$ is the greatest dimension of $\operatorname{rad} N$ for $N \in F_{m}(\boldsymbol{k})$. We recall that $s_{1}(\boldsymbol{R})=0$, $s_{m}(\boldsymbol{R})=1$ for $m>1$, and $s_{m}(\boldsymbol{C})$ is equal to the integer part of $m / 2$ (for the last assertion see [7], Theorem (3.11)). Let $N \in F_{m}(\boldsymbol{k})$ with $d=\operatorname{dim} N>0$. Denote $s=\operatorname{dim} \operatorname{rad} N$. Then $d+s \leqq m([7]$, Theorem 3.8) and there exists an orthogonal basis $\left\{e_{1}, \ldots, e_{d}\right\}$ for $N$ such that $\left\{e_{1}, \ldots, e_{s}\right\}$ is a basis for $\operatorname{rad} N$ if $s>0$ ([7], Theorems 3.3 and 3.7). Moreover, if $\boldsymbol{k}=\boldsymbol{R}$ and there exists an index $i \in\{s+1, \ldots, d\}$ such that $\left(e_{i}\right)^{2}>0$, then $\left(e_{j}\right)^{2}<0$ for any $j \neq i$.

In conformity with the above considerations, we shall introduce a natural set $E_{m}(\boldsymbol{k})$ consisting of certain orthogonal bases for all nonzero linear subspaces of $M_{m}(\boldsymbol{k})$. Let $E_{m d s}(\boldsymbol{k})$ [resp. $E_{m d+}(\boldsymbol{R})$ ] denote the set of all ordered bases $\underline{e}=\left(e_{1}, \ldots, e_{d}\right)$ for the $d$-dimensional linear subspaces of $M_{m}(\boldsymbol{k})$ [resp. $\left.M_{m}(\boldsymbol{R})\right]$ with $s$-dimensional (resp. 0-dimensional) radicals, which satisfy the orthogonality Conditions $(2.1),\left[\right.$ resp. $\left.\left(2.1^{\prime}\right)\right]$ given by

$$
\begin{aligned}
& e_{i} e_{j}=-\sum_{h=s+1}^{d} \delta_{i h} \delta_{j h}, \\
& e_{i} e_{j}=2 \delta_{i 1} \delta_{j 1}-\delta_{i j} ; \quad i, j=1, \ldots, d .
\end{aligned}
$$


We now introduce the following families of orthogonal bases:

$$
\begin{aligned}
& E_{m}(\boldsymbol{R})=\left(\bigcup_{d=1}^{m} E_{m d+}(\boldsymbol{R})\right) \cup\left(\bigcup_{d=1}^{m-1} \bigcup_{s=0}^{1} E_{m d s}(\boldsymbol{R})\right), \\
& E_{m}(\boldsymbol{C})=\bigcup_{d=1}^{m} \bigcup_{s=0}^{\min (d, m-d)} E_{m d s}(\boldsymbol{C}) .
\end{aligned}
$$

The sets $E_{m d+}(\boldsymbol{R})$ and $E_{m d s}(\boldsymbol{k})$ from the right side of (2.2) are called the Stiefel manifols for the Minkowski space.

Let $\theta_{m}: E_{m}(\boldsymbol{k}) \rightarrow F_{m}(\boldsymbol{k})$ be the mapping which carries every basis $\underline{e} \in E_{m}(\boldsymbol{k})$ to the linear space over $\boldsymbol{k}$ spanned by the vectors of $\underline{e}$. We write

$$
\begin{gathered}
F_{m d s}(\boldsymbol{k})=\theta_{m}\left(E_{m d s}(\boldsymbol{k})\right), \\
F_{m d+}(\boldsymbol{R})=\theta_{m}\left(E_{m d+}(\boldsymbol{R})\right) .
\end{gathered}
$$

Clearly the space $N \in F_{m}(\boldsymbol{R})$ of nonzero dimension $d$ belongs to $F_{m d+}(\boldsymbol{R})$ [resp. $F_{m d 0}(\boldsymbol{R})$, resp. $\left.F_{m d 1}(\boldsymbol{R})\right]$ if and only if there exists $x \in N$ with $(x)^{2}>0$ [resp. $x \in N, x \neq 0$, implies $(x)^{2}<0$, resp. $x \in N$ implies $(x)^{2} \leqq 0$ and there exists $x^{\prime} \in N, x^{\prime} \neq 0$, such that $\left.\left(x^{\prime}\right)^{2}=0\right]$.

Now let $F_{m d}(\boldsymbol{k})$ denote the Grassmann manifold of all $d$-dimensional linear subspaces of $M_{m}(\boldsymbol{k})$ [6]. Then the following relations hold:

$$
\begin{aligned}
F_{m}(\boldsymbol{k}) & =\theta_{m}\left(E_{m}(\boldsymbol{k})\right) \cup F_{m 0}(\boldsymbol{k}), \\
F_{m d}(\boldsymbol{R}) & =F_{m d+}(\boldsymbol{R}) \cup F_{m d 0}(\boldsymbol{R}) \cup F_{m d 1}(\boldsymbol{R}), \quad 1 \leqq d \leqq m-1, \\
F_{m d}(\boldsymbol{C}) & =\bigcup_{s=0}^{\min (d, m-d)} F_{m d s}(\boldsymbol{C}), \quad 1 \leqq d \leqq m .
\end{aligned}
$$

In order to describe the geometric structure of the Stiefel manifolds, we need certain groups of isometries.

A mapping $\Lambda$ from $N \in F_{m}(\boldsymbol{k})$ into $N^{\prime} \in F_{m}(\boldsymbol{k})$ is an isometry if $(\Lambda x)(\Lambda y)=x y$ for any $x, y \in N$. The full Lorentz group $O(1, m-1 ; \boldsymbol{k})$ consists of all isometries $\Lambda$ of $M_{m}(\boldsymbol{k})$ onto $M_{m}(\boldsymbol{k})$, where every $\Lambda$ is identified to the $m \times m$ matrix $\left(\Lambda_{v}^{\mu}\right), 0 \leqq \mu, v \leqq m-1$, defined by $(\Lambda x)^{\mu}=\sum_{\nu=0}^{m-1} \Lambda_{v}^{\mu} x^{\nu}$, $x \in M_{m}(\boldsymbol{k})$. According to this identification, the proper Lorentz group and the connected real Lorentz group are denoted by $S O(1, m-1 ; \boldsymbol{k})$ and $S O^{\uparrow}(1, m-1 ; \boldsymbol{R})$, respectively.

The full Lorentz group acts on $E_{m}(\boldsymbol{k})\left[\operatorname{resp} . F_{m}(\boldsymbol{k})\right]$ in the following way: $\Lambda\left(e_{1}, \ldots, e_{d}\right)=\left(\Lambda e_{1}, \ldots, \Lambda e_{d}\right)[\operatorname{resp} . \Lambda N=\{x \mid x=\Lambda y, y \in N\}]$ for any $\Lambda \in O(1, m-1 ; \boldsymbol{k})$ and $\left(e_{1}, \ldots, e_{d}\right) \in E_{m}(\boldsymbol{k})$ [resp. $\left.N \in F_{m}(\boldsymbol{k})\right]$.

It is clear that the Stiefel manifolds and their images under $\theta_{m}$ are Lorentz invariant. We will prove that these manifolds are homogeneous spaces of certain open subgroups of $O(1, m-1 ; \boldsymbol{k})$. 
Table 1

\begin{tabular}{|c|c|c|}
\hline$E$ & $G$ & $H$ \\
\hline$E_{m m+}(\boldsymbol{R})$ & $O(1, m-1 ; \boldsymbol{R})$ & $\{1\}$ \\
\hline $\begin{array}{l}E_{m, m-1,1}(\boldsymbol{R}) \\
m>1\end{array}$ & $O(1, m-1 ; \boldsymbol{R})$ & $\{1\}$ \\
\hline $\begin{array}{l}E_{m, m-1,+}(\boldsymbol{R}) \\
m>1\end{array}$ & $S O(1, m-1 ; \boldsymbol{R})$ & $\{1\}$ \\
\hline $\begin{array}{l}E_{m, m-1,0}(R) \\
m>1\end{array}$ & $S O(1, m-1 ; \boldsymbol{R})$ & $\{1\}$ \\
\hline $\begin{array}{l}E_{m d+}(\boldsymbol{R}) \\
1 \leqq d \leqq m-2\end{array}$ & $S O(1, m-1 ; \boldsymbol{R})$ & $S O(m-d ; \boldsymbol{R})$ \\
\hline $\begin{array}{l}E_{m d 1}(\bar{R}) \\
1 \leqq d \leqq m-2\end{array}$ & $S O(1, m-1 ; \boldsymbol{R})$ & $\boldsymbol{R}^{m-d-1} \odot S O(m-d-1 ; \boldsymbol{R})$ \\
\hline $\begin{array}{l}E_{m d 0}(\boldsymbol{R}) \\
1 \leqq d \leqq m-2\end{array}$ & $S O^{\dagger}(1, m-1 ; \boldsymbol{R})$ & $S O^{\dagger}(1, m-d-1 ; \boldsymbol{R})$ \\
\hline $\begin{array}{l}E_{m d s}(\bar{C}) \\
m / 2 \leqq d \leqq m \\
s=m-d\end{array}$ & $O(1, m-1 ; C)$ & $\begin{array}{ll}\{1\} & \text { if } s \leqq 1 \\
C^{s(s-1) / 2} & \text { if } s>1\end{array}$ \\
\hline $\begin{array}{l}E_{m d s}(C) \\
1 \leqq d \leqq m-1 \\
0 \leqq s \leqq \min (d, m-d-1)\end{array}$ & $S O(1, m-1 ; C)$ & {$\left[C^{s(m-d-s)} \odot S O(m-d-s ; C)\right] \bigcirc C^{s(s-1) / 2}$} \\
\hline
\end{tabular}

Table 2

\begin{tabular}{|c|c|c|}
\hline$F$ & $G^{\prime}$ & $H^{\prime}$ \\
\hline$F_{m m+}(R)$ & $S O^{\uparrow}(1, m-1 ; \boldsymbol{R})$ & $S O^{\dagger}(1, m-1 ; \boldsymbol{R})$ \\
\hline $\begin{array}{l}F_{m d+}(\boldsymbol{R}) \\
F_{m, m-d, 0}(\boldsymbol{R}) \\
1 \leqq d \leqq m-1\end{array}$ & $S O^{\uparrow}(1, m-1 ; \boldsymbol{R})$ & $\begin{array}{l}S O(m-1 ; \boldsymbol{R}) \text { if } d=1 \\
{\left[S O^{\dagger}(1, d-1 ; \boldsymbol{R}) \otimes S O(m-d ; \boldsymbol{R})\right] \odot O(1 ; \boldsymbol{R})} \\
\text { if } 1<d<m-1\end{array}$ \\
\hline $\begin{array}{l}F_{m d 1}(\boldsymbol{R}) \\
F_{m, m-d, 1}(\boldsymbol{R}) \\
1 \leqq d \leqq m-1\end{array}$ & $S O^{\dagger}(1, m-1 ; \boldsymbol{R})$ & $\begin{array}{l}\boldsymbol{R}_{+} \text {if } d=1 ; m=2 \\
{\left[\boldsymbol{R}^{m-2} \odot S O(m-2 ; \boldsymbol{R})\right] \otimes \boldsymbol{R}_{+}} \\
\text {if } d=1 ; m>2 \\
\left\{\left[\boldsymbol{R}^{d-1} \odot S O(d-1 ; \boldsymbol{R})\right]\right. \\
\left.\otimes\left[\boldsymbol{R}^{m-d-1} \odot S O(m-d-1 ; \boldsymbol{R})\right]\right\} \odot\left[O(1 ; \boldsymbol{R}) \otimes \boldsymbol{R}_{+}\right] \\
\text {if } 1<d<m-1\end{array}$ \\
\hline$F_{m m 0}(C)$ & $S O(1, m-1 ; C)$ & $S O(1, m-1 ; C)$ \\
\hline $\begin{array}{l}F_{m d 0}(C) \\
F_{m, m-d, 0}(C) \\
1 \leqq d \leqq m-1\end{array}$ & $S O(1, m-1 ; C)$ & $\begin{array}{l}S O(m-1 ; C) \text { if } d=1 \\
{[S O(d ; C) \otimes S O(m-d ; C)] \odot O(1 ; C)} \\
\text { if } 1<d<m-1\end{array}$ \\
\hline $\begin{array}{l}F_{m d s}(\boldsymbol{C}) \\
F_{m, m-d, s}(\boldsymbol{C}) \\
1 \leqq d \leqq m-1 \\
1 \leqq s \leqq \min (d, m-d)\end{array}$ & $S O(1, m-1 ; C)$ & $\begin{array}{l}C^{s(s-1) / 2} \mathrm{GL}(s ; C) \text { if } s=d=m / 2 \\
\left\{\left[C^{s(d-s)} \odot S O(d-s ; C)\right] \bigcirc\left[C^{s(s-1) / 2}\right\}\right. \\
\odot \mathrm{GL}(s ; C) \text { if } s=m-d, s<d \\
\left\{\left[C^{s(d-s)} \odot S O(d-s ; C)\right]\right. \\
\left.\otimes\left[C^{s(m-d-s)} \odot S O(m-d-s ; C)\right]\right\} \\
\left.\bigcirc C^{s(s-1) / 2}\right\} \odot[O(1 ; C) \otimes G L(s ; C)] \\
\text { if } s<\min (d, m-d)\end{array}$ \\
\hline
\end{tabular}


In order to avoid certain complications, we introduce Tables 1 and 2. Some explanations are required. $\operatorname{GL}(n ; \boldsymbol{k})$ denotes the group of all invertible $n \times n$ matrices whose coefficients belong to $\boldsymbol{k}$. The group $O(n ; \boldsymbol{k})[\mathrm{resp} . S O(n ; \boldsymbol{k})]$ consists of all orthogonal (resp. proper orthogonal) matrices of $\operatorname{GL}(n ; \boldsymbol{k})$. By an abuse of notation, both the $q$-dimensional Euclidean space over $\boldsymbol{k}$ and any Abelian Lie group isomorphic to the translation group of this space are denoted by $\boldsymbol{k}^{q}$. In Table $2, R_{+}$is the multiplicative group of all strictly positive real numbers. The symbols $\otimes_{0}$, and $\odot$ are reserved for the central extensions direct and semidirect products of groups, respectively. After these preparations, we prove an announced assertion.

Proposition 1. Let E, G, H, F, G', $H^{\prime}$ be as in Tables 1 and 2. Then $E$ (resp. $F$ ) is analytically isomorphic to $G / H$ (resp. $G^{\prime} / H^{\prime}$ ).

In Proposition 1 and throughout this paper, "analytic" means "real analytic" if $\boldsymbol{k}=\boldsymbol{R}$ and "complex analytic" if $\boldsymbol{k}=\boldsymbol{C}$.

Proof. With no loss of generality we may suppose that $\theta_{m}(E)=F$.

1) We first establish that $G$ (resp. $G^{\prime}$ ) acts transitively on $E$ (resp. $F$ ).

Let $\underline{e}=\left(e_{1}, \ldots, e_{d}\right) \in E_{m}(\boldsymbol{k})$. Denote $N=\theta_{m}(\underline{e})$ and $s=\operatorname{dim} \operatorname{rad} N$.

A completion of $\underline{e}$ is an ordered basis $\left(e_{1}, \ldots, e_{m}\right)$ for $M_{m}(\boldsymbol{k})$ satisfying the following properties:

a) if $s>0$, then $e_{i} e_{m-s+j}=\delta_{i j}$ for all $i=1, \ldots, m$ and $j=1, \ldots, s$;

b) if $m-d-s>0$, then $e_{i} e_{d+j}=\alpha_{j} \delta_{i, d+j}$ for all $i=1, \ldots, m$ and $j=1, \ldots, m-d-s$, where: 1) $\alpha_{j}=-1$ for $j>1$; 2) $\alpha_{1}^{2}=1$; 3) $\alpha_{1}=1$ if and only if $\underline{e} \in E_{m d 0}(\boldsymbol{R})$.

We recall that there exists at least one completion of $\underline{e}([7]$, Theorems 3.5 and 3.8).

Assume that $\left(e_{1}, \ldots, e_{m}\right)$ is a completion of $\underline{e}$. Consider an arbitrary basis $\underline{e}^{\prime}=\left(e_{1}^{\prime}, \ldots, e_{d}^{\prime}\right) \in E$ having the completion $\left(e_{1}^{\prime}, \ldots, e_{m}^{\prime}\right)$. It now follows from the Properties a) and b) that $e_{i} e_{j}=e_{i}^{\prime} e_{j}^{\prime}$ for any $i, j=1, \ldots, m$. Then the linear transformation $\Lambda$ of $M_{m}(\boldsymbol{k})$, defined by $\Lambda e_{i}=e_{i}^{\prime}$ for all $i=1, \ldots, m$, is an isometry. Obviously $\underline{e}^{\prime}=\Lambda \underline{e}$. Therefore $O(1, m-1 ; \boldsymbol{k})$ acts transitively on $E$. The transitive action of $O(1, m-1 ; \boldsymbol{k})$ on $F$ is automatically induced by $\theta_{m}$.

Consider now the involutions $\Lambda_{i} \in O(1, m-1 ; \boldsymbol{k})$ defined by $\Lambda_{i} e_{j}$ $=\left(1-2 \delta_{i j}\right) e_{j}$ for all $i, j=1, \ldots, m$. Let $L_{i_{1} \ldots i_{q}}$ be the subgroup of $O(1, m-1 ; \boldsymbol{k})$ generated by $\Lambda_{i_{1}}, \ldots, \Lambda_{i_{q}}$, where $\left\{i_{1}, \ldots, i_{q}\right\} \subset\{1, \ldots, m\}$. Plainly $L_{i_{1} \ldots i_{q}} \underline{e}=\{\underline{e}\}$ (resp. $L_{i_{1} \ldots i_{q}} \cdot N=\{N\}$ ) for any $\left\{i_{1}, \ldots, i_{q}\right\} \subset\{d+1$, $\ldots, m-d-s\}$ (resp. $\left.\left\{i_{1}, \ldots, i_{q}\right\} \subset\{1, \ldots, m\}\right)$. Then $O(1, m-1 ; \boldsymbol{k})$ is isomorphic to $S O(1, m-1 ; \boldsymbol{k}) \odot L_{m}$. Moreover, $O(1, m-1, \boldsymbol{R})$ is isomorphic to $S O^{\uparrow}(1, m-1 ; \boldsymbol{R}) \odot L_{12}$ (resp. $\left.S O^{\uparrow}(1, m-1 ; \boldsymbol{R})\right) \odot L_{h, d+1}$ if $\underline{e} \in E_{m d+}(\boldsymbol{R})$ $\cup E_{m d 1}(\boldsymbol{R})$ (resp. $e \in E_{m d 0}(\boldsymbol{R}), h=1$ or $h=m>d+1$ ). On the other hand, it is easy to see that if $L$ is a normal subgroup of $O(1, m-1 ; \boldsymbol{k})$ such that 
$L \underline{e}=\{\underline{e}\}($ resp. $L N=\{N\})$, then the group $O(1, m-1 ; \boldsymbol{k}) / L$ acts transitively on $E$ (resp. $F$ ). These results imply immediately that $S O(1, m-1 ; \boldsymbol{k})$ acts transitively on $E$ if $d+s<m$ (and also on $F$ ). Moreover, if $k \in \boldsymbol{R}$, then $S O^{\dagger}(1, m-1 ; \boldsymbol{R})$ acts transitively on $E$ if $\underline{e} \in E_{m d 0}(\boldsymbol{R}), d<m-1$ (and also on $F$ ).

2) We next compute the little group $H_{0}$ of $\underline{e}$ and the little group $H_{0}^{\prime}$ of $N$ defined by

$$
\begin{aligned}
& H_{0}=\{\Lambda \mid \Lambda \in O(1, m-1 ; \boldsymbol{k}), \Lambda \underline{e}=\underline{e}\}, \\
& H_{0}^{\prime}=\{\Lambda \mid \Lambda \in O(1, m-1 ; \boldsymbol{k}), \Lambda N=N\} .
\end{aligned}
$$

We shall prove that $H=G \cap H_{0}$ and $H^{\prime}=G^{\prime} \cap H_{0}^{\prime}$. Consider $\Lambda \in H_{0}^{\prime}$. Define the $m \times m$ matrix $\tilde{\Lambda}=\left(\tilde{\Lambda}_{j}^{i}\right), 1 \leqq i, j \leqq m$, by $\Lambda e_{j}=\sum_{h=1}^{m} \tilde{\Lambda}_{h}^{i} e_{i}$. Simple computations show that the conditions $\Lambda N=N$ and $\left(\Lambda e_{i}\right)\left(\Lambda e_{j}\right)=e_{i} e_{j}$, where $i, j=1, \ldots, m$, are equivalent to the following relations:

$$
\begin{aligned}
& \tilde{\Lambda}=\left(\begin{array}{llll}
B_{11} & B_{12} & B_{13} & B_{14} \\
0 & B_{22} & 0 & B_{24} \\
0 & 0 & B_{33} & B_{34} \\
0 & 0 & 0 & B_{44}
\end{array}\right) \begin{array}{l}
d-s \\
s-d-s \\
s
\end{array} \\
& B_{h h}^{t} g_{(h)} B_{h h}=g_{(h)}, \quad B_{h 4}=-B_{h h} g_{(h)} B_{1 h}^{t}\left(B_{11}^{t}\right)^{-1}, \quad h=2,3 \text {, } \\
& B_{14}=-\frac{1}{2}\left(B+\sum_{h=2}^{3} B_{1 h} g_{(h)} B_{1 h}^{t}\right)\left(B_{11}^{t}\right)^{-1}, \quad B_{44}=\left(B_{11}^{t}\right)^{-1} \text {, }
\end{aligned}
$$

where $B$ is a skew-symmetric $s \times s$ matrix whose coefficients belong to $\boldsymbol{k}$, the $(d-s) \times(d-s)$ [resp. $(m-d-s) \times(m-d-s)]$ matrix $g_{(2)}$ (resp. $g_{(3)}$ ) is defined by $g_{(2) i j}=e_{s+i} e_{s+j}$ for any $i, j=1, \ldots, d-s$ (resp. $g_{(3) i j}=e_{d+i} e_{d+j}$ for any $\left.i, j=1, \ldots, m-d-s\right)$. 0 denotes the zero matrix and the symbol $t$ denotes the matrix transpose. The $0 \times q$ or $q \times 0$ blocks from (2.6) are omitted.

By (2.6), the coefficients of $B_{h 4}, h=1,2,3,4$, are rational functions of the coefficients of the other blocks. Write $\tilde{A}=\left(B_{12}, B_{22}, B_{13}, B_{33}, B\right.$, $\left.B_{11}\right)$. The the composition rule in $H_{0}^{\prime}$ is $\tilde{\Lambda}^{\prime \prime}=\tilde{\Lambda} \tilde{\Lambda}^{\prime}$ with

$$
\begin{aligned}
B_{h h}^{\prime \prime} & =B_{h h} B_{h h}^{\prime}, \quad h=1,2,3, \\
B_{1 h}^{\prime \prime} & =B_{11} B_{1 h}^{\prime}+B_{1 h} B_{h h}^{\prime}, \quad h=2,3, \\
B^{\prime \prime} & =B+B_{11} B^{\prime} B_{11}^{t}+\sum_{h=2}^{3}\left(B_{1 h} B_{h h}^{\prime} g_{(h)} B_{1 h}^{\prime t} B_{11}^{t}-B_{11} B_{1 h}^{\prime} g_{(h)} B_{h h}^{t} B_{1 h}^{t}\right) .
\end{aligned}
$$

The parameter blocks generate the following groups: $\left\{B_{12}\right\}=\boldsymbol{k}^{s(d-s)}$, $\left\{B_{22}\right\}=K, \quad\left\{B_{13}\right\}=\boldsymbol{k}^{s(m-d-s)}, \quad\left\{B_{33}\right\}=K^{\prime},\{B\}=\boldsymbol{k}^{s(s-1) / 2}, \quad\left\{B_{11}\right\}=\mathrm{GL}$ 
$(s ; \boldsymbol{k})$, where $K$ (resp. $\left.K^{\prime}\right)$ is the group of all matrices $B_{22} \in \mathrm{GL}(d-s ; k)$ [resp. $\left.B_{33} \in \mathrm{GL}(m-d-s ; k)\right]$ which satisfy the relation $B_{22}^{t} g_{(2)} B_{22}=g_{(2)}$ (resp. $\left.B_{33}^{t} g_{(3)} B_{33}=g_{(3)}\right)$. It follows from the definition of $\left(e_{1}, \ldots, e_{m}\right)$ that $K$ is isomorphic to $O(1, d-1 ; \boldsymbol{R})$ [resp. $O(d ; \boldsymbol{R})$, resp. $O(d-s ; \boldsymbol{k})]$ and $K^{\prime}$ is isomorphic to $O(m-d ; \boldsymbol{R}) \quad[\operatorname{resp} . O(1, m-d-1 ; \boldsymbol{R})$, resp. $O(m-d-s ; \boldsymbol{k})]$ if $\underline{e}$ belongs to $E_{m d+}(\boldsymbol{R})\left[\operatorname{resp} . E_{m d 0}(\boldsymbol{R})\right.$, resp. $E_{m d s}(\boldsymbol{k})$ with either $\boldsymbol{k}=\boldsymbol{R}$ and $s=1$ or $\boldsymbol{k}=\boldsymbol{C}$ ].

By (2.7), the following isomorphism holds:

$$
H_{0}^{\prime} \rightarrow\left\{\left[\left(\boldsymbol{k}^{s(d-s)} \odot K\right) \otimes\left(\boldsymbol{k}^{s(m-d-s)} \odot K^{\prime}\right) \bigcirc \boldsymbol{k}^{s(s-1) / 2}\right]\right\} \odot \mathrm{GL}(s ; \boldsymbol{k}) .
$$

The group $H_{0}$ is a normal subgroup of $H_{0}^{\prime}$. If $\tilde{\Lambda} \in H_{0}$, then it follows from $\tilde{\Lambda} \underline{e}=\underline{e}$ that $B_{11}$ and $B_{22}$ are unit matrices and $B_{12}=0$. Then (2.8) induces the following isomorphism:

$$
H_{0} \rightarrow\left(\boldsymbol{k}^{s(m-d-s)} \odot K^{\prime}\right) \bigcirc \boldsymbol{k}^{s(s-1) / 2} .
$$

The assertion of this part of the proof follows straight-forward from (2.8) and (2.9), and the foregoing results.

3) Finally, we consider that $E$ is canonically embedded in $\boldsymbol{k}^{d m}$

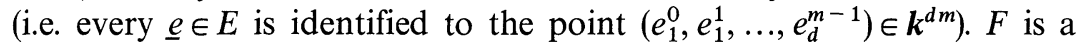
subset of $F_{m d}(\boldsymbol{k})$. The Grassmann manifold $F_{m d}(\boldsymbol{k})$ is an analytic manifold of dimension $d(m-d)$ [6]. The rest of the proof is standard. $H$ (resp. $\left.H^{\prime}\right)$ is a Lie subgroup of $G$ (resp. $G^{\prime}$ ) and the homogeneous space $G / H$ (resp. $G^{\prime} / H^{\prime}$ ) is an analytic manifold. The orbit $E=G \underline{e}$ (resp. $F=G N$ ) of $G$ is an analytic submanifold of $\boldsymbol{k}^{d m}\left[\mathrm{resp} . F_{m d}(\boldsymbol{k})\right]$ isomorphic to $G / H$ (resp. $G^{\prime} / H^{\prime}$ ) with respect to the natural map $g \underline{e} \rightarrow g H(\operatorname{resp} . g N \rightarrow g H$ ), $g \in G$ ([8], Part. II, Chapter IV, $\S 5$, Theorems 1, 3, 4, and their Consequence).

The following remarks improve Proposition 1:

Remark 1. The manifolds $E_{m d 0}(\boldsymbol{R}), 1 \leqq d \leqq m-2$, and $E_{m d s}(\boldsymbol{C})$, $1 \leqq d \leqq m-1,0 \leqq s \leqq \min (d, m-d-1)$, are connected. Consider $E$ as in Table 1. Let $S E$ be the set of all $\underline{e} \in E$ with $\operatorname{det}\left(e_{i}^{\mu}\right)>0$, where $\left(e_{1}, \ldots, e_{m}\right)$ is a completion of $\underline{e}$ and $i=1, \ldots, m ; \mu=0,1, \ldots, m-1$. Write $I E=E \backslash S E$. If $\boldsymbol{k}=\boldsymbol{R}$, then $E^{\uparrow}$ denotes the set of all $e \in E$ with $e_{1}^{0}>0$. Denote $E^{\downarrow}=E \backslash E^{\uparrow}$, $S E^{\uparrow}=S E \cap E^{\uparrow}, S E^{\downarrow}=S E \cap E^{\downarrow}, I E^{\uparrow}=I E \cap E^{\uparrow}$, and $I E^{\downarrow}=I E \cap E^{\downarrow}$. The manifold $E \in\left\{E_{m m+}(\boldsymbol{R}), E_{m, m-1}(\boldsymbol{R})\right\}$ has four connected components: $S E^{\uparrow}, S E^{\downarrow}, I E^{\uparrow}$, and $I E^{\downarrow}$. The manifold $E \in\left\{E_{m, m-1,+}(\boldsymbol{R}), E_{m, m-1,0}(\boldsymbol{R})\right.$, $\left.E_{m d+}(\boldsymbol{R}), E_{m d 1}(\boldsymbol{R})\right\}, 1 \leqq d \leqq m-2$, has two connected components: $E^{\uparrow}$ and $E^{\downarrow}$. The manifold $E \in\left\{E_{m d s}(\boldsymbol{C})\right\}, m / 2 \leqq d \leqq m, s=m-d$, has two connected components $S E$ and $I E$.

Consider now $E$ and $\underline{e} \in E$ as in the proof of Proposition 1. Since the vectors $e_{1}, \ldots, e_{d}$ are linearly independent, there exists a permutation $\left\{\mu_{1}, \mu_{2}, \ldots, \mu_{m}\right\}$ of $\{0,1, \ldots, m-1\}$ such that all determinants $\operatorname{det}\left(e_{i}^{\mu_{j}}\right)_{1 \leqq i, j \leqq h}$; 
$h \leqq d$, are not zero in a neighbourhood $V(\underline{e})$ of $\underline{e}$ in $E$. A straightforward calculation show that $\left\{e_{i}^{\mu_{j}}\right\}, i \in\{1, \ldots, d\} ; \mu_{j} \in\{0,1, \ldots, m-1\} \backslash\left\{\mu_{1}, \ldots, \mu_{i}\right\}$, is a system consisting of $m d-d(d+1) / 2$ local coordinates in $V(\underline{e})$. This result gives a concrete analytic structure of the manifold $E$ of codimension $d(d+1) / 2$ in $\boldsymbol{k}^{d m}$. Moreover, $\bar{E}$ is a $d(2 m-d-1) / 2-$ dimensional algebraic subvariety of $\boldsymbol{k}^{d m}$. Here the symbol ".-" is used for the closure of sets. If $s=0$, then $E$ is closed. If $s>0$, then $E$ is dense and open in $\bar{E}$ and the boundary of $E$ can be decomposed into a disjoint union of manifolds isomorphic to the union of the manifolds $E_{m d^{\prime} s^{\prime}}(\boldsymbol{k})$ with $d^{\prime}<d$ and $s^{\prime}=d^{\prime}+s-d$.

Remark 2. All manifolds $F$ from Table 2 are connected. Consider, $F, \underline{e}$, and $N$ as in Remark 1 and the proof of Proposition 1. Define a basis $\left\{f_{1}, \ldots, f_{d}\right\}$ for $N$ such that $f_{i}=\Sigma_{j=1}^{d} a_{i j} e_{j},\left(a_{i j}\right) \in \mathrm{GL}(d ; \boldsymbol{k})$, where $f_{i}^{\mu_{j}}=\delta_{i j}$, $i, j=1, \ldots, d$, and $\left(\mu_{1}, \ldots, \mu_{m}\right)$ is a permutation of $(0, \ldots, m-1)$. Then $\left\{f_{i}^{\mu_{j}}\right\}, i=1, \ldots, d ; j=d+1, \ldots, m$, is a system of local coordinates in a neighbourhood of $N$ in $F_{m d}(\boldsymbol{k})$. Introduce the Gram matrix $P=\left(f_{i} f_{j}\right)$, $1 \leqq i, j \leqq d$. It is easy to see that $\operatorname{dim} \operatorname{rad} N=s$ if and only if rank $P=d-s$. The set of all symmetric $d \times d$ matrices of rank $d-s$ is an analytic manifold of codimension $s(s+1) / 2$ in the manifold of all symmetric $d \times d$ matrices. Then $F$ is an analytic submanifold of $F_{m d}(\boldsymbol{k})$ of dimension $d(m-d)-s(s+1) / 2$. Since the mapping $\theta_{m}$ is open (i.e. $\theta_{m}$ carries open sets onto open sets) the boundary properties from Remark 1 persist if $E$ and $\bar{E}$ are replaced by $F$ and $\bar{F}$, respectively.

\section{The Variety of Scalar Products}

In this section we discuss the connection between the product of $n$ Minkowski spaces and its image on the variety of scalar products. We begin with some notation and conventions.

Let $M_{m}^{n}(\boldsymbol{k})$ denote the topological product of $n$ Minkowski spaces $M_{m}(\boldsymbol{k}) . M_{m}^{n}(\boldsymbol{k})$ consists of all points $(x)_{n}=\left(x_{1}, \ldots, x_{n}\right)$, where $x_{i} \in M_{m}(\boldsymbol{k})$, $i=1, \ldots, n$. For any $(x)_{n} \in M_{m}^{n}(\boldsymbol{k})$, let $[x]_{n}$ be the $n \times m$ matrix whose coefficients are $x_{i}^{\mu}(i=1, \ldots, n ; \mu=0,1, \ldots, m-1)$. The Lorentz group acts on $M_{m}^{n}(\boldsymbol{k})$ by $\Lambda(x)_{n}=\left(\Lambda x_{1}, \ldots, \Lambda x_{n}\right)$ for any $(x)_{n} \in M_{m}^{n}(\boldsymbol{k})$ and $\Lambda \in 0(1, m-1 ; \boldsymbol{k})$.

We now present some geometric properties of the mapping $\pi$ which carries each point of $M_{m}^{n}(\boldsymbol{k})$ to its Gram matrix. Many remarkable properties of this mapping have been established in Refs. [2, 3] and [9].

Let $S_{n}(\boldsymbol{k})$ denote the set of all symmetric $n \times n$ matrices whose coefficients belong to $\boldsymbol{k}$. $S_{n}(\boldsymbol{k})$ is identified with $\boldsymbol{k}^{n(n+1) / 2}$ carrying each matrix $Z \in S_{n}(k)$ into the point of coordinates $Z_{i j}(1 \leqq i \leqq j \leqq n)$, where $Z_{i j}, 1 \leqq i, j \leqq n$, are the coefficients of $Z$. 
It is convenient to define the following minors of $Z \in S_{n}(\boldsymbol{k})$ :

$$
\begin{aligned}
& G_{i_{1}}^{j_{1} \ldots j_{h}}(Z)=\operatorname{det}\left(Z_{i j}\right)_{i=i_{1}}, \ldots, i_{h} ; j=j_{1}, \ldots, j_{h} \\
& G_{i_{1} \ldots i_{h}}(Z)=G_{i_{1} \ldots i_{h}}^{i_{1} \ldots i_{h}}(Z),
\end{aligned}
$$

where $h, i_{1}, \ldots, i_{h}, j_{1}, \ldots, j_{h} \in\{1, \ldots, n\}$. For any $Z \in S_{n}(\boldsymbol{R})$ denote

$$
\varepsilon_{i_{1} \ldots i_{h}}(Z)=\operatorname{sgn}(-1)^{h-1} G_{i_{1} \ldots i_{h}}(Z),
$$

where $\operatorname{sgn} \alpha=1$ if $\alpha>0, \operatorname{sgn} \alpha=0$ if $\alpha=0$, and $\operatorname{sgn} \alpha=-1$ if $\alpha<0$ for any $\alpha \in \boldsymbol{R}$.

The mapping $\pi$ from $M_{m}^{n}(\boldsymbol{k})$ into $S_{n}(\boldsymbol{k})$ is defined by $Z=\pi\left((x)_{n}\right)$ with $Z_{i j}=x_{i} x_{j}$ for any $(x)_{n} \in M_{m}^{n}(\boldsymbol{k})$ and $i, j=1, \ldots, n$. The set $\pi\left(M_{m}^{n}(\boldsymbol{k})\right)$ is called the variety of scalar products.

$S_{n r}(\boldsymbol{k})$ denotes the set of all matrices $Z \in \pi\left(M_{m}^{n}(\boldsymbol{k})\right)$ with rank $Z=r$. Let $S_{n r+}(\boldsymbol{R})$ [resp. $S_{n r-}(\boldsymbol{R})$ ] be the set of all matrices belonging to $S_{n r}(\boldsymbol{R})$ with only one positive eigenvalue (resp. only negative eigenvalues).

We shall now give a constructive proof of the following proposition:

Proposition 2. a) Let $1 \leqq r \leqq \min (m, n)$. There exist a finite open covering $\left\{S_{n r \gamma}(\boldsymbol{k})\right\}_{\gamma \in \Gamma_{n r}}$ of the analytic manifold $S_{n r}(\boldsymbol{k})$ and a holomorphic mapping $\sigma_{n r \gamma}$ from $S_{n r \gamma}(\boldsymbol{k})$ into $M_{m}^{n}(\boldsymbol{k})$ with $\pi \sigma_{n \boldsymbol{r} \gamma}=1$ for any $\gamma \in \Gamma_{n \boldsymbol{r}}$.

b) The following relations hold:

$$
\begin{aligned}
\pi\left(M_{m}^{n}(\boldsymbol{k})\right) & =\bigcup_{r=0}^{\min (m, n)} S_{n r}(\boldsymbol{k}), \\
S_{n m}(\boldsymbol{R}) & =S_{n m+}(\boldsymbol{R}), \quad n \geqq m, \\
S_{n r}(\boldsymbol{R}) & =S_{n r+}(\boldsymbol{R}) \cup S_{n r-}(\boldsymbol{R}), \quad 1 \leqq r \leqq \min (m-1, n),
\end{aligned}
$$

$\pi\left(M_{m}^{n}(\boldsymbol{C})\right)$ is the algebraic variety of all matrices $Z \in S_{n}(\boldsymbol{C})$ such that $\operatorname{rank} Z \leqq \min (m, n) . \pi\left(M_{m}^{n}(\boldsymbol{R})\right)$ is the semi-algebraic variety of all matrices $Z \in S_{n}(\boldsymbol{R})$ such that:

1) $\operatorname{rank} Z \leqq \min (m, n): 2) \varepsilon_{i_{1} \ldots i_{m}}(Z) \geqq 0$ if $\operatorname{rank} Z=m$ and $\left\{i_{1}, \ldots, i_{m}\right\}$ $C\{1, \ldots, n\}$; 3) the relation

$$
\varepsilon_{i_{1} \ldots i_{h}}(Z)>\varepsilon_{i_{1} \ldots i_{h+1}}(Z)
$$

implies $G_{i_{1} \ldots i_{h+1}}(Z)=0$ for $h, i_{1}, \ldots, i_{h+1} \in\{1, \ldots n\}$. Moreover $Z \in S_{n r-}(\boldsymbol{R})$ if and only if $Z \in S_{n r}(R), 1 \leqq r \leqq \min (m-1, n)$, and $\varepsilon_{i_{1} \ldots i_{h}}(Z) \leqq 0$ for any $h, i_{1}, \ldots i_{h} \in\{1, \ldots, n\}$.

Proof. a) Suppose that $1 \leqq r \leqq \min (m, n)$. Let $S_{n r 1}(\boldsymbol{k})$ denote the set of all $Z \in S_{n r}(\boldsymbol{k})$ such that $G_{1 \ldots h}(Z) \neq 0$ for any $h \in\{1, \ldots, r\}$. 
We shall construct a finite set $\Gamma_{n r}$ of orthogonal $n \times n$ matrices such that $S_{n r}(\boldsymbol{k})$ is spanned by $\gamma^{t} Z \gamma$, where $Z \in S_{n r 1}(\boldsymbol{k})$ and $\gamma \in \Gamma_{n r}$. In order to do this, we introduce some notations.

Let $\Pi_{n}$ denote the image of the group of all permutations of $(1, \ldots, n)$ under its isomorphism which carries each permutation $\left(i_{1}, \ldots, i_{n}\right)$ of $(1, \ldots, n)$ into the $n \times n$ matrix $\tau=\left(\tau_{j j^{\prime}}\right)$ with $\tau_{j j^{\prime}}=\delta_{i_{j} j^{\prime}} ; j, j^{\prime}=1, \ldots, n$. It is obvious that $\left(\tau Z \tau^{t}\right)_{j j^{\prime}}=Z_{i_{j} i_{j^{\prime}}}$ for any $Z \in S_{n}(\boldsymbol{k})$.

$\Pi_{n r}$ (resp. $\Pi_{n p}^{\prime}$ with $\left.p \in\{2, \ldots, r\}\right)$ denotes the subset of $\Pi_{n}$ consisting of all matrices corresponding to all permutations $\left(i_{1}, \ldots, i_{n}\right)$ of $(1, \ldots n)$ with $i_{h}<i_{h+1}$ if $h \neq r$ (resp. with: 1) $i_{h}<i_{h+1}$ if $h \leqq p-3 ; 2$ ) either $i_{p-2}<i_{p-1}$ or $\left.i_{p-1}<i_{p} ; 3\right) i_{h}=h$ if $h>p$ ).

Define the $n \times n$ matrix $\omega_{p}=\left(\omega_{p i j}\right)$ by

$$
\begin{aligned}
\omega_{p i j}= & \delta_{i j}+2^{-1 / 2}\left(\delta_{i, p-1} \delta_{j p}-\delta_{i p} \delta_{j, p-1}\right) \\
& +\left(2^{-1 / 2}-1\right)\left(\delta_{i p} \delta_{j p}+\delta_{i, p-1} \delta_{j, p-1}\right),
\end{aligned}
$$

where $i, j=1, \ldots, n$ for any fixed $p \in\{2, \ldots, r\}$.

Let $\Gamma_{n r}$ denote the set of all orthogonal matrices

$$
\gamma=\omega_{2}^{\alpha_{2}} \tau_{2} \omega_{3}^{\alpha_{3}} \tau_{3} \ldots \omega_{r}^{\alpha_{r}} \tau_{r} \tau
$$

where $\tau \in \Pi_{n r}, \tau_{p}=\Pi_{n p}^{\prime}, p=2, \ldots, r$, and $\alpha_{p}=0,1\left(\omega_{p}^{0}\right.$ is the unit $n \times n$ matrix and $\left.\omega_{p}^{1}=\omega_{p}\right)$.

Let $Z \in S_{n r}(\boldsymbol{k})$. We prove that there exists $\gamma \in \Gamma_{n r}$ such that $\gamma Z \gamma^{t} \in S_{n r 1}(\boldsymbol{k})$. Consider $\gamma$ as in (3.6).

Define $Z_{p-1}=\omega_{p}^{\alpha_{p}} \tau_{p} Z_{p} \tau_{p}^{t}\left(\omega_{p}^{\alpha_{p}}\right)^{t}$ and $Z_{r}=\tau Z \tau^{t}$, where $p=2, \ldots, r$.

Since $Z \in S_{n r}(\boldsymbol{k})$, there exists a nonzero diagonal minor $G_{i_{1} \ldots i_{r}}(Z)$ with $i_{1}<\cdots<i_{r}$ (see, for example, Ref. [6] from [2]). Choose $\tau \in \Pi_{n r}$ corresponding to the permutation $\left(i_{1}, \ldots, i_{n}\right)$ of $(1, \ldots, n)$ with $i_{r+1}<\cdots<i_{n}$. Then $G_{1 \ldots r}\left(Z_{r}\right) \neq 0$.

Consider now the following inductive hypothesis:

$$
I_{p}: G_{1 \ldots h}\left(Z_{p}\right) \neq 0 \text { for } h=p, \ldots, r .
$$

It is obvious that $I_{r}$ holds. Let $p \in\{2, \ldots, r\}$ and suppose that $I_{p}$ holds. Then there are only two cases:

Case $A$. There exists a nonzero diagonal minor $G_{j_{1} \ldots j_{p-1}}\left(Z_{p}\right)$ with $j_{1}<\cdots<j_{p-1} \leqq p$

Case $B$. Case A is not satisfied, but there exists a non-zero minor $G_{j_{1} \ldots j_{p}-2 j_{p}}^{j_{1} \ldots j_{p-2}}\left(Z_{p}\right)$ with $j_{1}<\cdots<j_{p-2} \leqq p$ and $j_{p-1}<j_{p} \leqq p$.

Choose $\tau_{p} \in \Pi_{n p}^{\prime}$ corresponding to the permutation $\left(j_{1}, \ldots, j_{p-1}, j_{p}\right.$, $p+1, \ldots, n)$. Using in Case B the identity

$$
\begin{aligned}
2 G_{1 \ldots p-1}\left(\omega_{p} Z^{\prime} \omega_{p}^{t}\right)= & G_{1 \ldots p-1}\left(Z^{\prime}\right) \\
& +G_{1 \ldots p-2 p}\left(Z^{\prime}\right)+2 G_{1 \ldots p-2 p}^{1 \ldots p-2 p-1}\left(Z^{\prime}\right)
\end{aligned}
$$


for $Z^{\prime}=\tau_{p} Z_{p} \tau_{p}^{t}$, it follows that $G_{1 \ldots p-1}\left(Z_{p-1}\right) \neq 0$. Clearly this result is also satisfied in Case A. Choosing $\alpha_{p}=0$ in Case A and $\alpha_{p}=1$ in Case B, $I_{p-1}$ holds. By induction on $p, I_{1}$ holds. Hence $Z_{1}=\gamma Z \gamma^{t} \in S_{n r 1}(\boldsymbol{k})$ for some $\gamma \in \Gamma_{n r}$.

We now construct the complex analytic structure of $S_{n r}(\boldsymbol{k})$. Fix $\gamma \in \Gamma_{n r}$ and set $S_{n r \gamma}(k)=\gamma^{t} S_{n r 1}(k) \gamma$. Let $Z_{0} \in S_{n r \gamma}(\boldsymbol{k})$ and set $Z_{0}^{\prime}=\gamma Z_{0} \gamma^{t}$. Since $Z_{0}^{\prime} \in S_{n r 1}(\boldsymbol{k})$, there exists a neighbourhood $V \subset S_{n}(\boldsymbol{k})$ of $Z_{0}$ such that $G_{1 \ldots h}\left(\gamma Z \gamma^{t}\right) \neq 0$ for any $Z \in V$ and $h=1, \ldots, r$.

We can define the following coordinate system of $V$ :

$$
\eta_{i j}=\left(\gamma Z \gamma^{t}\right)_{i j}, \quad \eta_{k l}=G_{1 \ldots r k}^{1 \ldots r l}\left(\gamma Z \gamma^{t}\right)
$$

where $1 \leqq i \leqq j \leqq n, i \leqq r, r<k \leqq l \leqq n$, and $Z \in V$. Indeed, the Jacobian of the transformation of Coordinates (3.8) is non-vanishing on $V$ because it is equal to $\left(G_{1 \ldots r}\left(\gamma Z \gamma^{t}\right)\right)^{(n-r)(n-r+1) / 2}$. Moreover, since the rank of any matrix belonging ot $V$ is not smaller than $r$, the set $V \cap S_{n r}(\boldsymbol{k})$ consists of all $Z \in V$ with $\eta_{k l}(Z)=0$ for any $k, l \in\left\{r_{+1}, \ldots, n\right\}, k \leqq l$. Then $S_{n r}(\boldsymbol{k})$ is a complex analytic submanifold of $S_{n}(\boldsymbol{k})$ of codimension $(n-r)$ $\cdot(n-r+1) / 2$. with

Define the holomorphic mapping $\sigma_{n r \gamma}: S_{n r}(\boldsymbol{k}) \rightarrow M_{m}^{n}(\boldsymbol{k})$ by $\sigma_{n r \gamma}(Z)=(x)_{n}$

$$
\begin{aligned}
& x_{i}^{\prime \mu_{1}}=Z_{i 1}^{\prime}\left(\chi_{1} Z_{11}^{\prime}\right)^{-1 / 2}, \quad x_{i}^{\prime \mu_{J}}=0 \\
& x_{i}^{\prime \mu_{h}}=G_{1 \ldots h-1 h}^{1 \ldots h-1 i}\left(Z^{\prime}\right)\left[\chi_{h} G_{1 \ldots h-1 h-1}\left(Z^{\prime}\right) G_{1 \ldots h}\left(Z^{\prime}\right)\right]^{-1 / 2},
\end{aligned}
$$

where $Z \in S_{n r \gamma}(\boldsymbol{k}), Z^{\prime}=\gamma Z \gamma^{t},[x]_{n}=\gamma^{t}\left[x^{\prime}\right]_{n}, h=2, \ldots, r$, and $j=r+1, \ldots, m$. Here $\chi_{1}=1, \chi_{h}=-1$ if $k=C$ and $\chi_{1}=\varepsilon_{1}\left(Z^{\prime}\right), \chi_{h}=\varepsilon_{1 \ldots h-1}\left(Z^{\prime}\right) \varepsilon_{1 \ldots h}\left(Z^{\prime}\right)$ if $\boldsymbol{k}=\boldsymbol{R}$. $\left(\mu_{1}, \ldots, \mu_{m}\right)$ is a permutation of $(0,1, \ldots, m-1)$ which satisfies the following conditions: 1) $\mu_{h}=h-1$ if $k=\boldsymbol{C}$;2) if $\boldsymbol{k}=\boldsymbol{R}$ and $\mu_{h}=0$, then either $h=r+1$ or $\varepsilon_{1 \ldots h-1}\left(Z^{\prime}\right) \varepsilon_{1 \ldots h}\left(Z^{\prime}\right)=-1$ with the convention $\varepsilon_{1 \ldots h-1}\left(Z^{\prime}\right)=-1$ for $\left.h=1 ; 3\right)$ if $\boldsymbol{k}=\boldsymbol{R}, h<h^{\prime}$, and $\mu_{h}, \mu_{h^{\prime}} \neq 0$, then $\mu_{h}<\mu_{h^{\prime}}$. The above choice of $\left(\mu_{1}, \ldots, \mu_{m}\right)$ is permitted according to Jacobi's theorem ([10], Chapter X, Theorem 2): if $Z^{\prime} \in S_{n r+}(R)$ [resp. $\left.Z^{\prime} \in S_{n r-}(R)\right]$ and $G_{1 \ldots h}\left(Z^{\prime}\right) \neq 0, h=1, \ldots, r$, then $\varepsilon_{1 \ldots h-1}\left(Z^{\prime}\right) \varepsilon_{1 \ldots h}\left(Z^{\prime}\right)=1$ for $r-1$ indices $h \in\{1, \ldots, r\}$ (resp. for any $h=1, \ldots, r$ ).

Indeed, it suffices to prove the last Relation (3.3). If $Z^{\prime} \in \pi\left(M_{m}^{n}(\boldsymbol{k})\right)$, then there exists $(y)_{n} \in M_{m}^{n}(\boldsymbol{k})$ such that $[y]_{n}(g)_{m}[y]_{n}^{t}=Z^{\prime}$, where $g_{(m)}=\left(g_{(m) \mu v}\right), 0 \leqq \mu, v \leqq m-1$, is a diagonal $m \times m$ matrix with $g_{(m) 00}=1$ and $g_{(m) \mu \mu}=-1$ for $\mu>0$. Then $\operatorname{rank} Z^{\prime} \leqq \operatorname{rank}[y]_{n} \leqq \min (m, n)$, Moreover, in the case $\boldsymbol{k}=\boldsymbol{R}$, since $(g)_{m}$ has only one positive eigenvalue, it follows from Silvester's law of inertia that $Z^{\prime}$ has at most one positive eigenvalue (if rank $Z^{\prime}=m$, then $Z^{\prime}$ has only one positive eigenvalue). Thus (3.3) holds. 
The branches of the square roots from (3.9) are taken to have nonnegative real parts.

Straightforward computations starting from (3.9) show that $[x]_{n} g_{(m)}[x]_{n}^{t}=Z$ (see, for example, the Gauss algorithm from [10], Chapter II, §4). Therefore $\pi\left(\sigma_{n r \gamma}(Z)\right)=Z$ for any $Z \in S_{n r \gamma}(\boldsymbol{k})$ and a) is proved.

b) According to the proof of a), (3.3) holds. Let $A(\boldsymbol{k})$ [resp. $B(\boldsymbol{k})]$ denote the set of all matrices $Z \in \pi\left(M_{m}^{n}(\boldsymbol{k})\right)$ [resp. $Z \in S_{n}(\boldsymbol{k})$ with 1] rank $Z \leqq \min (m, n)$; 2) $\varepsilon_{i_{1} \ldots i_{h}}(Z) \leqq \varepsilon_{i_{1} \ldots i_{h+1}}(Z)$ if $\boldsymbol{k}=\boldsymbol{R}, i_{1}, \ldots, i_{h+1} \in\{1, \ldots, n\}$, $h<\operatorname{rank} Z$; 3) $\varepsilon_{i_{1} \ldots i_{m}}(Z) \geqq 0$ if $\boldsymbol{k}=\boldsymbol{R}, i_{1}, \ldots, i_{m} \in\{1, \ldots, n\}$, rank $Z=m$ ) such that $G_{i_{1} \ldots i_{h}}(Z) \neq 0$ if $i_{1}, \ldots, i_{h} \in\{1, \ldots, n\}, i_{1}<\cdots<i_{h}$ and $h \leqq$ rank $Z$. Let $B_{n r-}(\boldsymbol{R})$ be the set of all $Z \in B(\boldsymbol{R})$ such that rank $Z=r$ and $\varepsilon_{i_{1} \ldots i_{h}}(Z) \leqq 0$ for any $i_{1}, \ldots, i_{h} \in\{1, \ldots, n\}$. It follows from the proof of a) that $A(\boldsymbol{k})=B(\boldsymbol{k})$ and $A(\boldsymbol{R}) \cap S_{n r-}(\boldsymbol{R})=B_{n r-}(\boldsymbol{R})$. Since $A(\boldsymbol{k})$ is dense in $\pi\left(M_{m}^{n}(\boldsymbol{k})\right)$, we have $\pi\left(M_{m}^{n}(\boldsymbol{k})\right)=\bar{B}(\boldsymbol{k}), \quad S_{n r-}(\boldsymbol{R})=\bar{B}_{n \boldsymbol{r}-}(\boldsymbol{R}) \cap\left\{Z \mid Z \in S_{n}(\boldsymbol{R})\right.$, rank $\left.Z=r\right\}$ and b) holds. We recall that a semialgebraic variety can be realized as a subset of a real Euclidean space locally defined by a system of poliynomial equations and inequalities (see, for example, [11], Chapter I, § 3).

Properties 2) and 3) from Proposition 2b) give the polynomial inequalities for $\pi\left(M_{m}^{n}(\boldsymbol{R})\right)$, but the polynomial equations are given by Property 1) [i.e. by the vanishing of all Gram minors of order greater than $\min (m, n)]$.

Remark 3. The manifolds $S_{n r^{+}}(\boldsymbol{R}), S_{n r^{\prime}-}$ and $S_{n r}(\boldsymbol{C})$ with $1 \leqq r \leqq \min (m, n)$ and $1 \leqq r^{\prime} \leqq \min (m-1, n)$ are nonvoid and connected. Indeed, $Z \in S_{n r+}(\boldsymbol{R})$ [resp. $Z \in S_{n r^{\prime}-}(\boldsymbol{R})$, resp. $\left.Z \in S_{n r}(\boldsymbol{C})\right]$ if and only if $Z=O D O^{t}$, where $O \in S O(n ; \boldsymbol{R})[$ resp. $O \in S O(n ; \boldsymbol{R})$, resp. $O \in S U(n ; \boldsymbol{C})]$ and $D=\left(d_{i j}\right)$ is a diagonal $n \times n$ matrix with $d_{i i} \in \boldsymbol{R}$ (resp. $d_{i i} \in \boldsymbol{R}$, resp. $d_{i i} \in \boldsymbol{C}$ ), $i=1, \ldots, n$, and $d_{11}>0, d_{i i}<0$ for $1<i \leqq r, d_{i i}=0$ for $i>r$ (resp. $d_{i i}<0$ for $i \leqq r^{\prime}$, $d_{i i}=0$ for $i>r^{\prime}$, resp. $d_{i i} \neq 0$ for $i \leqq r, d_{i i}=0$ for $i>r$ ). It suffices to remark that $S O(n ; \boldsymbol{k})$ and the set spanned by $D$ are connected, and the mapping $(O, D) \rightarrow O D O^{t}$ is continuous.

We next list certain useful properties of $S_{n r}(\boldsymbol{k}), S_{n r}(\boldsymbol{k})$ is open and dense in $\bar{S}_{n r}(\boldsymbol{k}) . \bar{S}_{n r}(\boldsymbol{C})$ [resp. $\bar{S}_{n r}(\boldsymbol{R}), \bar{S}_{n r \pm}(\boldsymbol{R})$ ] is an algebraic (resp. semialgebraic) subvariety of $S_{n}(\boldsymbol{C})$ [resp. $\left.S_{n}(\boldsymbol{R})\right]$. If $r>0, \bar{S}_{n, r-1}(\boldsymbol{k})$ is the boundary (and also the singular locus) of $\bar{S}_{n r}(\boldsymbol{k})$. The boundary of $S_{n r+}(\boldsymbol{R}) \quad$ [resp. $\left.S_{n r^{\prime}-}(\boldsymbol{R})\right], 1 \leqq r \leqq m$ (resp. $\left.1 \leqq r^{\prime}<m\right)$, is $\bar{S}_{n, r-1}(\boldsymbol{R})$ [resp. $\left.\bar{S}_{n, r^{\prime}-1}(\boldsymbol{R})\right]$. The boundary of $S_{n 1-}(\boldsymbol{R})$ is the set $S_{n 0}(\boldsymbol{R})$ consisting only of the zero matrix. Notice that $\bar{S}_{n \min (m, n)}(\boldsymbol{k})=\pi\left(M_{m}^{n}(\boldsymbol{k})\right)$. A standard proof of these results can be extracted from [11] (see especially Chapter I, 2.4, Propositions 2 and 3).

Notice that the Gram determinantal constraints given by Proposition $2 \mathrm{~b}$ ) for $m=4$ and $\boldsymbol{k}=\boldsymbol{R}$ generalize Byers' and Yang's 
results of relativistic multiparticle kinematics [12] (several details and applications can be found in [13]).

In order to describe the inverse image (under $\pi$ ) of any Gram matrix belonging to $\pi\left(\mathrm{M}_{m}^{n}(\boldsymbol{k})\right)$, we write a fundamental lemma of $\mathrm{Hall}$ and Wightman [2] in the following form:

Lemma 1. a) Let $Z \in \pi\left(\left(M_{m}^{n}(\boldsymbol{k})\right)\right)$ and $r=\operatorname{rank} Z$. Then any $(x)_{n} \in \pi^{-1}(Z)$ satisfies the following decomposition:

$$
x_{i}=\sum_{j=d-r+1}^{d} \alpha_{i j} e_{j}+\sum_{h=1}^{d-r} \beta_{i h} e_{h} \quad i=1, \ldots, n,
$$

where $\underline{e}=\left(e_{1}, \ldots, e_{d}\right) \in E_{m}(\boldsymbol{k}), \beta=\left(\beta_{i h}\right)$ with $i=1, \ldots, n$ and $h=1, \ldots d-r$ is an $n \times(d-r)$ matrix of rank $d-r$ (in the case $d>r$ ), and the $n \times r$ matrix $\alpha=\left(\alpha_{i j}\right)$ is chosen such that $\alpha_{i j}=\xi_{i}^{v_{j}} ; i=1, \ldots n ; j=d-r+1, \ldots, d$, $(\xi)_{n}=\sigma_{n r \gamma}(Z), Z \in S_{n r \gamma}(\boldsymbol{k}), \gamma \in \Gamma_{n r} ; v_{j} \in\{0,1, \ldots, m-1\}, v_{j}<v_{j^{\prime}}$ for $j<j^{\prime}$, and $\xi_{i}^{v_{j}} \neq 0$ for some $i$ at any fixed $j$ (in the case $r>0$ ). The first (resp. the second) sum in the r.h.s. of (3.10) is dropped if $r=0$ (resp. $r=d$ ). Moreover, $Z \in S_{n r+}(\boldsymbol{R})$ (resp. $Z \in S_{n r-}(\boldsymbol{R})$, resp. $Z \in S_{n r}(\boldsymbol{C})$ ) if and only if $\underline{e} \in E_{m r+}(\boldsymbol{R})$ (resp. $\underline{e} \in E_{m r 0}(\boldsymbol{R}) \cup E_{m d 1}(\boldsymbol{R})$, resp. $\underline{e} \in E_{m d, d-r}(\boldsymbol{C})$ ).

b) Let $\left(x^{\prime}\right)_{n} \in M_{m}^{n}(\boldsymbol{k})$ and let $(x)_{n}$ be as in a). Then there exists a Lorentz transformation $\Lambda \in O(1, m-1 ; \boldsymbol{k})$ such that $\left(x^{\prime}\right)_{n}=\Lambda(x)_{n}$ if and only if there exist an orthogonal basis $\underline{e}^{\prime}=\left(e_{1}^{\prime}, \ldots, e_{d}^{\prime}\right) \in E_{m}(\boldsymbol{k})$ with $\left(e_{j}^{\prime}\right)^{2}=\left(e_{j}\right)^{2}$, $j=1, \ldots, d$, an $r \times(d-r)$ matrix $\Delta$ whose coefficients belong to $\boldsymbol{k}$, and an invertible $(d-r) \times(d-r)$ matrix $\Omega \in \mathrm{GL}(d-r ; \boldsymbol{k})$ such that $(3.10)$ holds for $(x)_{n}, \underline{e}$, and $\beta$ replaced by $\left(x^{\prime}\right)_{n}, \underline{e}^{\prime}$, and $\alpha \Delta+\beta \Omega$ respectively.

Proof. a) We shall use the notation of Lemma 1 a). Let $N$ denote the linear space over $\boldsymbol{k}$ spanned by $x_{1}, \ldots, x_{n}$. Then we can write $x_{i}=y_{i}+z_{i}$, $i=1, \ldots, n$, where $z_{i} \in \operatorname{rad} N$ and $y_{i} \in N$ is orthogonal to $\operatorname{rad} N$ for any $\mathrm{i}$ ([7], Theorem 3.3). Let $N_{1}$ (resp. $N_{2}$ ) be the linear space over $\boldsymbol{k}$ spanned by $y_{1}, \ldots, y_{n}$ (resp. $\left.\xi_{1}, \ldots, \xi_{n}\right)$. Since $\pi\left((y)_{n}\right)=\pi\left((\xi)_{n}\right)=Z$, the isometry $\Lambda^{\prime}: N_{2} \rightarrow N_{1}$ defined by $\Lambda^{\prime} \xi_{i}=y_{i}, i=1, \ldots, n$, can be extended to a Lorentz transformation $\Lambda \in O(1, m-1 ; \boldsymbol{k})$ (as in the proof of Proposition 1).

If $r>0$, we introduce the orthogonal basis $f=\left(f_{1}, \ldots, f_{r}\right) \in E_{m}(\boldsymbol{k})$ defined by

$$
\xi_{i}=\sum_{j=d-r+1}^{d} \alpha_{i j} f_{j}, \quad i=1, \ldots, n .
$$

Set $e_{j}=\Lambda f_{j}, j=d-r+1, \ldots, d$. If $d>r$, there exists an orthogonal basis $\left(e_{1}, \ldots, e_{d-r}\right) \in E_{m, d-r, d-r}(\boldsymbol{k})$ for $\operatorname{rad} N$ such that

$$
z_{i}=\sum_{h=1}^{d-r} \beta_{i h} e_{h}, \quad i=1, \ldots, n ; \beta_{i h} \in \boldsymbol{k} .
$$


By (3.11) and (3.12), we obtain (3.10). By (3.9), $f \in E_{m r+}(\boldsymbol{R})$ [resp. $\left.f \in E_{m r 0}(\boldsymbol{R}), f \in E_{m r 0}(\boldsymbol{C})\right]$ if and only if $Z \in S_{n r+}(\boldsymbol{R})$ [resp. $Z \in S_{n r-}(\boldsymbol{R})$, $\left.Z \in S_{n r}(\boldsymbol{C})\right]$. Using the relations $E_{m r+}(\boldsymbol{R})=\Lambda E_{m r+}(\boldsymbol{R})$ and $E_{m r 0}(\boldsymbol{k})$ $=\Lambda E_{m r 0}(\boldsymbol{k})$, assertion a) follows immediately.

b) We shall use the notations of Lemma $1 \mathrm{~b}$ ). Suppose first that $\left(x^{\prime}\right)_{n}=\Lambda(x)_{n}$. Set $e_{j}^{\prime}=\Lambda e_{j}, \Delta=0$, and $\Omega=1$. Then (3.10) holds for $(x)_{n}$ and $\underline{e}$ replaced by $\left(x^{\prime}\right)_{n}$ and $\underline{e}^{\prime}$, respectively.

Suppose next that (3.10) holds for $(x)_{n}, \underline{e}$, and $\beta$ replaced by $\left(x^{\prime}\right)_{n}, \underline{e}^{\prime}$, and $\alpha \Delta+\beta \Omega$, respectively. According to the proof of Proposition 1, we can define a Lorentz transformation $\Lambda \in O(1, m-1 ; \boldsymbol{k})$ such that:

$$
\begin{aligned}
& \Lambda e_{j}=e_{j}^{\prime}+\sum_{h=1}^{d-r} \Delta_{j h} e_{h}^{\prime}, \quad j=d-r+1, \ldots, d, \\
& \Lambda e_{h}=\sum_{h^{\prime}=1}^{d-r} \Omega_{h h^{\prime}} e_{h^{\prime}}^{\prime}, \quad h=1, \ldots, d-r .
\end{aligned}
$$

By (3.13), we have $\Lambda(x)_{n}=\left(x^{\prime}\right)_{n}$ and b) holds.

Remark 4. According to the proof of Proposition 2b), the matrix $\alpha$ in (3.10) is uniquely and completely determined by $Z$ provided $\gamma$ is fixed. In this case, the Decomposition (3.10) with $d=r$ is unique.

Suppose that $d>r$ and denote $s=d-r$. Denote by $Q_{m d s}(Z, k)$ the quotient space of the set of all pairs $(\underline{e}, \beta)$, where $\underline{e} \in E_{m d s}(\boldsymbol{k})$ and $\beta$ is an $n \times s$ matrix of rank $s$ whose coefficients belong to $\boldsymbol{k}$, with respect to the following equivalence relation: $(e, \beta)$ is equivalent to $\left(e^{\prime}, \beta^{\prime}\right)$ if and only if (3.13) holds with $\Delta$ and $\Omega$ as in Lemma $1 \mathrm{~b}$ ) and $\Lambda e_{j}=e_{j}^{\prime}, j=1, \ldots, d$. The equivalence class of $(e, \beta)$ is isomorphic to the group $\{\Delta, \Omega\}=\boldsymbol{k}^{r s} \odot \mathrm{GL}$ $(s ; \boldsymbol{k})$ and has a representative $q=\left(\underline{e}^{\prime}, \beta^{\prime}\right)$ such that $\beta_{i_{j} h}^{\prime}=\delta_{j+r h}, j=1, \ldots, d$; $h=1, \ldots, s$, for some $i_{r+1}, \ldots, i_{d} \in\{1, \ldots, n\}$ and for $i_{1}, \ldots, i_{r}$ chosen as in (3.9). Then $Q_{m d s}\left(Z^{\prime}, \boldsymbol{k}\right)$ can be identified with the analytic manifold $E_{m d s}(\boldsymbol{k}) \times F_{n-r, s}(\boldsymbol{k})$, where $F_{n-r, s}(\boldsymbol{k})$ is the Grassmann manifold of all $s$-dimensional linear subspaces of $\boldsymbol{k}^{n-\boldsymbol{r}}$, provided $Z^{\prime}$ belongs to a neighbourhood of $Z$ in $S_{n r}(\boldsymbol{k})$ such that (3.9) holds. $F_{n-r, s}(\boldsymbol{k})$ is realized as the quotient space of all $(n-r) \times s$ matrices $\beta=\left(\beta_{i h}\right)$ of rank $s, \beta_{i h} \in \boldsymbol{k}$, $i \in\{1, \ldots, n\} \backslash\left\{i_{1}, \ldots, i_{r}\right\}, h=1, \ldots, s$, with respect to $\operatorname{GL}(s ; \boldsymbol{k})$.

Notice that (3.10) is uniquely determined by a representative $\left(e^{\prime}, \beta^{\prime}\right)$ of the equivalence class of $(e, \beta)$ such that the $i_{1}, \ldots, i_{r}$ th lines of $\beta^{\prime}$ are vanishing.

Let $M_{m d s}^{n}(\boldsymbol{k})\left[\operatorname{resp} . M_{m d+}^{n}(\boldsymbol{R})\right]$ denote the set of all points $(x)_{n} \in M_{m}^{n}(\boldsymbol{k})$ $\left[\right.$ resp. $\left.(x)_{n} \in M_{m}^{n}(\boldsymbol{R})\right]$ such that there exists a basis belonging to $E_{m d s}(\boldsymbol{k})$ $\left[\right.$ resp. $\left.E_{m d+}(\boldsymbol{R})\right]$ for the linear space over $\boldsymbol{k}$ spanned by $x_{1}, \ldots, x_{n}$. This linear space is $d$-dimensional and its radical is $s$-dimensional. Denote by $M_{m 00}^{n}(\boldsymbol{k})$ the set consisting of the only zero point $(0)_{n} \in M_{m}^{n}(k)$. 
By (2.2) and Lemma 1, we have

$$
\begin{aligned}
& M_{m}^{n}(\boldsymbol{R})=M_{m 00}^{n}(\boldsymbol{R}) \cup\left(\bigcup_{d=1}^{\min (m, n)} M_{m d+}^{n}(\boldsymbol{R})\right) \cup\left(\bigcup_{d=1}^{\min (m-1, n)} \bigcup_{s=0}^{1} M_{m d s}^{n}(R)\right) \\
& M_{m}^{n}(\boldsymbol{C})=\bigcup_{d=0}^{\min (m, n)} \bigcup_{s=0}^{\min (m-d, d)} M_{m d s}^{n}(\boldsymbol{C}) \\
& \pi\left(M_{m d+}^{n}(\boldsymbol{R})\right)=S_{n d+}(\boldsymbol{R}), \quad 1 \leqq d \leqq \min (m, n), \\
& \pi\left(M_{m d 0}^{n}(\boldsymbol{R})\right)=S_{n d-}(\boldsymbol{R}), \quad 1 \leqq d \leqq \min (m-1, n) \\
& \pi\left(M_{m d 1}^{n}(\boldsymbol{R})\right)=S_{n, d-1,-}(\boldsymbol{R}), \quad 1 \leqq d \leqq \min (m-1, n) \\
& \pi\left(M_{m 00}^{n}(\boldsymbol{R})\right)=S_{n 0}(\boldsymbol{R}), \\
& \pi\left(M_{m d s}^{n}(\boldsymbol{C})\right)=S_{n, d-s}(\boldsymbol{C}), \quad 0 \leqq d \leqq \min (m, n), \quad 0 \leqq s \leqq \min (d, m-d)
\end{aligned}
$$

where $S_{n 0-}(\boldsymbol{R})=S_{n 0}(\boldsymbol{R})$.

Remark 5. We now list certain useful properties of $M_{m d+}^{n}(\boldsymbol{R})$ and $M_{m d s}^{n}(\boldsymbol{k})$ which follow from Proposition 2, Lemma 1, Remarks 1-4, (3.14), and (3.15). If $Z \in S_{n r+}(\boldsymbol{R})$ [resp. $Z \in S_{n r-}(\boldsymbol{R})$, resp. $Z \in S_{n r}(\boldsymbol{C})$ ], then there exists a neighbourhood $V$ of $Z$ in $S_{n r+}(\boldsymbol{R})$ [resp. $S_{n r-}(\boldsymbol{R})$, resp. $\left.S_{n r}(C)\right]$ Such that the following analytic isomorphisms induced by (3.10) hold: $\pi^{-1}(V) \cap M_{m r+}^{n}(\boldsymbol{R}) \sim V \times E_{m r+}(\boldsymbol{R})\left[\right.$ resp. $\pi^{-1}(V) \cap M_{m r 0}^{n}(\boldsymbol{R})$ $\sim V \times E_{m r 0}(\boldsymbol{R}), \pi^{-1}(V) \cap M_{n, r+1,1}^{n}(\boldsymbol{R}) \sim V \times E_{m, r+1,1}(\boldsymbol{R}) \times F_{n-r, 1}(\boldsymbol{R})$, resp. $\left.\pi^{-1}(V) \cap M_{m, r+s, s}^{n}(\boldsymbol{C}) \sim V \times E_{m, r+s, s}(\boldsymbol{C}) \times F_{n-r, s}(\boldsymbol{C})\right]$. Since $\operatorname{dim} F_{n-r, s}(\boldsymbol{k})$ $=s(n-r-s)$ and $\operatorname{rank} \pi$ is constant on $V, M_{m d+}^{n}(\boldsymbol{R})\left[\operatorname{resp} . M_{m d s}^{n}(\boldsymbol{k})\right]$ is an analytic submanifold of $M_{m}^{n}(\boldsymbol{R})\left[\mathrm{resp} . M_{m}^{n}(\boldsymbol{k})\right]$ of dimension $d(m+n-d)$ [resp. $d(m+n-d)-s(s+1) / 2]$ with respect to the structure induced by the preceding isomorphisms. The manifold $M_{m d+}^{n}(\boldsymbol{R})$ [resp. $\left.M_{m d s}^{n}(\boldsymbol{k})\right]$ is connected excepting the case $1 \leqq d=n \leqq m$ (resp. $\boldsymbol{k}=\boldsymbol{R} . s=1,1 \leqq d$ $=n \leqq m) ; M_{m d+}^{d}(\boldsymbol{R})$ [resp. $\left.M_{m d 1}^{d}(\boldsymbol{R})\right]$ has two connected components defined by sgn $e_{1}^{0}$ in (3.10). $M_{m d^{+}}^{n}(\boldsymbol{R})$ and $M_{m d s}^{n}(\boldsymbol{k})$ are open and dense in their closures. $\bar{M}_{m d+}^{n}(\boldsymbol{R})$, or $\bar{M}_{m d s}^{n}(\boldsymbol{R}), s=0,1$, [resp. $\left.\bar{M}_{m d s}^{n}(\boldsymbol{C})\right]$ is a semialgebraic (resp. algebraic) subvariety of $M_{m}^{n}(\boldsymbol{R})$ [resp. $\left.M_{m}^{n}(\boldsymbol{C})\right]$. The boundary of $M_{m d+}^{n}(\boldsymbol{R})\left[\right.$ resp. $M_{m d 0}^{n}(\boldsymbol{R})$, resp. $\left.M_{m d 1}^{n}(\boldsymbol{R})\right]$ is $\bar{M}_{m, d-1,+}^{n}(\boldsymbol{R})$ $\cup \bar{M}_{m d 1}^{n}(\boldsymbol{R})$ for $d<m$ and $\bar{M}_{m, m-1,+}^{n}(\boldsymbol{R}) \cup M_{m, m-1,0}^{n}(\boldsymbol{R})$ for $d=m$ [resp. $\bar{M}_{m d 1}^{n}(\boldsymbol{R})$, resp. $\left.\bar{M}_{m, d-1,0}^{n}(\boldsymbol{R})\right]$. The boundary of $\boldsymbol{M}_{m d s}^{n}(\boldsymbol{k})$ consists of the union of all $M_{m d^{\prime} s^{\prime}}^{n}(\boldsymbol{k})$ with $d^{\prime} \leqq d, s^{\prime} \geqq s$, and $d^{\prime}+s^{\prime}<d+s$.

$A$ stratification of the set $M \subset \boldsymbol{k}^{p}$ is a finite collection $\left\{M_{j}\right\}_{j \in J}$ of subsets of $M$, called strata, such that:

1) any stratum $A_{j}$ is a connected analytic submanifold of $\boldsymbol{k}^{p}$ open and dense in its closure $\bar{A}_{j} ; \bar{A}_{j}$ is a semialgebraic (resp. algebraic) subvariety of $\boldsymbol{k}^{p}$ for $\boldsymbol{k}=\boldsymbol{R}$ (resp. $\boldsymbol{k}=\boldsymbol{C}$ ) whose boundary $\bar{A}_{j} \backslash A_{j}$ is the union of the strata $A_{j^{\prime}}$, where $j^{\prime} \in J^{\prime}, \operatorname{dim} A_{j}^{\prime}<\operatorname{dim} A_{j}$, and $J^{\prime}$ is a subset of $J$ dependent of $j$;2) $A$ is the union of all strata $A_{j}, j \in J$. 
Let $\tilde{M}_{m}^{n}(\boldsymbol{R})\left[\right.$ resp. $\left.\tilde{M}_{m}^{n}(\boldsymbol{C})\right]$ be the set of all connected components of $M_{m d^{+}}^{n}(\boldsymbol{R}), M_{m d^{\prime} 0}^{n}(\boldsymbol{R})$, and $M_{m d^{\prime \prime} 1}^{n}(\boldsymbol{R}), 1 \leqq d \leqq \min (m, n), 0 \leqq d^{\prime} \leqq \min (m-1, n)$, $1 \leqq d^{\prime \prime} \leqq \min (m-1, n) \quad\left[\right.$ resp. $M_{m d s}^{n}(\boldsymbol{C}), 0 \leqq d \leqq \min (m, n), 0 \leqq s \leqq \min$ $(d, m-d)]$. Then $\tilde{M}_{m}^{n}(\boldsymbol{k})$ is a stratification of $M_{m}^{n}(\boldsymbol{k})$. Notice that the set of the images under $\pi$ of the strata of $M_{m}^{n}(\boldsymbol{k})$ is a stratification of $\pi\left(M_{m}^{n}(\boldsymbol{k})\right)$ [i.e. the set $\tilde{S}_{n \min (m, n)}(\boldsymbol{k})$ of all connected components of $S_{n r}(\boldsymbol{k}), 0 \leqq r \leqq \min$ $(m, n)$, is a stratification of $\pi\left(M_{m}^{n}(\boldsymbol{k})\right.$ and the inverse image under $\pi$ of any its stratum is a finite union of strata of $\tilde{M}_{m}^{n}(\boldsymbol{k})$ given by (3.15)].

A triplet $(M, \pi, S)$ is called an analytic fibre bundle if $M$ and $S$ are analytic manifolds, $\pi$ is an analytic mapping from $M$ onto $S$, and there exists an analytic manifold $T$ (called typical fibre) such that for any open set $V \subset S$ the set $\pi^{-1}(V)$ is analytically isomorphic to $V \times T$.

According to the foregoing results and definitions, we have

Theorem 1. Let $M \in \tilde{M}_{m}^{n}(\boldsymbol{k})$ and $S=\pi(M)$. Then $(M, \pi, S)$ is an analytic fibre bundle whose typical fibre is a connected component of $T$ given by Table 3 provided $M \subset M^{\prime}$.

Remark 6. The preceding algebraic results persist if $\boldsymbol{R}$ (resp. $\boldsymbol{C}$ ) is replaced by an ordered field whose positive elements are squares (resp. a field of characteristic zero whose elements are squares). If this field is a complete nondiscrete valued one, then the preceding analytic

Table 3

\begin{tabular}{llll}
\hline$M^{\prime}$ & $S$ & $T$ & $W$ \\
\hline $\begin{array}{l}M_{m d+}^{n}(\boldsymbol{R}) \\
1 \leqq d \leqq \min (m, d)\end{array}$ & $S_{n d+}(\boldsymbol{R})$ & $E_{m d+}(\boldsymbol{R})$ & $\{1\}$ \\
$M_{m d 0}^{n}(\boldsymbol{R})$ & & & \\
$1 \leqq d \leqq \min (m-1, n)$ & $S_{n d-}(\boldsymbol{R})$ & $E_{m d 0}(\boldsymbol{R})$ & $\{1\}$ \\
$M_{m d 1}^{n}(\boldsymbol{R})$ & & & $\{1\}$ \\
$n \leqq d \leqq m-1$ & $S_{n, d-1,-}(\boldsymbol{R})$ & $E_{m d 1}(\boldsymbol{R})$ & \\
$M_{m d 1}^{n}(\boldsymbol{R})$ & & & \\
$1 \leqq d \leqq \min (m-1, n-1)$ & $S_{n, d-1,-}(\boldsymbol{R})$ & $E_{m d 1}(\boldsymbol{R}) \times F_{n-d+1,1}(\boldsymbol{R})$ & $F_{n-d+1,1}(\boldsymbol{R})$ \\
$\begin{array}{l}M_{m 00}^{n}(\boldsymbol{R}) \\
M_{m d s}^{n}(\boldsymbol{C})\end{array}$ & $S_{n 0}(\boldsymbol{R})$ & $\{1\}$ & $\{1\}$ \\
$\begin{array}{l}1 \leqq d \leqq \min (m, n) \\
S(d-n) \leqq 0\end{array}$ & $S_{n, d-s}(\boldsymbol{C})$ & $E_{m d s}(\boldsymbol{C})$ & $\{1\}$ \\
$0 \leqq s \leqq \min (d, m-d)$ & & & \\
$M_{m d s}^{n}(\boldsymbol{C})$ & & & \\
$1 \leqq d \leqq \min (m-1, n-1)$ & $S_{n, d-s}(\boldsymbol{C})$ & $E_{m d s}(\boldsymbol{C}) \times F_{n-d+s, s}(\boldsymbol{C})$ & $F_{n-d+s, s}(\boldsymbol{C})$ \\
$1 \leqq s \leqq \min (d, m-d)$ & & & $\{1\}$ \\
$M_{m 00}^{n}(\boldsymbol{C})$ & $S_{n 0}(\boldsymbol{C})$ & $\{1\}$ & \\
\hline
\end{tabular}


results also persist. Notice that $s_{m}(\boldsymbol{k})=1$ [resp. $s_{m}(\boldsymbol{k})$ is the integer part of $m / 2$ ] if and only if $\boldsymbol{k}$ is ordered (resp. -1 is a square in $\boldsymbol{k}$ ).

\section{The Lorentz Orbit Space}

We now digress a little into the geometrical significance of certain natural sets of orbits of the Lorentz group $O(1, m-1 ; \boldsymbol{k})$ acting on the topological product of $n$ Minkowski spaces $M_{m}^{n}(\boldsymbol{k})$.

The orbit of $O(1, m-1 ; \boldsymbol{k})$ which contains the point $(x)_{n} \in M_{m}^{n}(\boldsymbol{k})$ is the set $O(1, m-1 ; \boldsymbol{k})(x)_{n}$ of all points $\Lambda(x)_{n}=\left(\Lambda x_{1}, \ldots, \Lambda x_{n}\right)$, where $\Lambda \in O(1, m-1 ; \boldsymbol{k})$.

Let $P$ be a Lorentz invariant subset of $M_{m}^{n}(\boldsymbol{k})$. Then $P$ is a union of orbits. Let $P / O(1, m-1 ; \boldsymbol{k})$ denote the quotient space of $P$ with respect to the following equivalence relation: $(x)_{n} \in P$ is equivalent to $(y)_{n} \in P$ if (and only if) $(y)_{n} \in O(1, m-1 ; \boldsymbol{k})(x)_{n} . P$ is considered with its relative topology as a subspace of $M_{m}^{n}(\boldsymbol{k})$. The quotient space (of Lorentz orbits) $P / O(1, m-1 ; \boldsymbol{k})$ is endowed with the quotient topology: a subset of $P / O(1, m-1 ; \boldsymbol{k})$ is open if and only if its inverse image under the canonical mapping $\left.\varrho\right|_{P}: P \rightarrow P / O(1, m-1 ; k)$ is open, where $\left.\varrho\right|_{P}$ is the restriction to $P$ of the open mapping $\varrho: M_{m}^{n}(\boldsymbol{k}) \rightarrow M_{m}^{n}(\boldsymbol{k}) / O(1, m-1 ; \boldsymbol{k})$ which carries any $(x)_{n} \in M_{m}^{n}(\boldsymbol{k})$ to its orbit $O(1, m-1 ; \boldsymbol{k})(x)_{n}$. The topological space $M_{m}(\boldsymbol{k}) / O(1, m-1 ; \boldsymbol{k})$ is called the Lorentz orbit space. This space is not a Hausdorff one. Indeed, it is easy to see that $O(1, m-1 ; \boldsymbol{k})(x)_{n}$ and $O(1, m-1 ; \boldsymbol{k})(y)_{n}$ are disjoint orbits with nonvoid intersection of their closures for $\left(x_{1}\right)^{2}=0, x_{1} \neq 0$, and $x_{2}=\cdots=x_{n}=y_{1}=\cdots=y_{n}=0$.

The image of any stratum of $M_{m}^{n}(\boldsymbol{k})$ under $\varrho$ is a Hausdorff subspace of the Lorentz orbit space homeomorphic to a connected analytic manifold. Thus if $M \in \tilde{M}_{m}^{n}(\boldsymbol{k})$ and $M \subset M^{\prime}$ with $M^{\prime}$ given in Table 3, then $\varrho(M)$ is homeomorphic to $S \times W$, where $W=T / O(1, m-1 ; \boldsymbol{k})$ with $S, T$, and $W$ given by Theorem 1 and Table 3 . Notice that $M^{\prime}$ is invariant with respect to $O(1, m-1 ; \boldsymbol{k})$. Let $(x)_{n} \in M^{\prime}$. The Lorentz orbit $O(1, m-1 ; \boldsymbol{k})(x)_{n}$ is completely determined by the Gram matrix $\pi\left((x)_{n}\right) \in S$ and an element of the Grassmann manifold $W$ (if $\operatorname{dim} W>0$ ). This orbit is homeomorphic to the Stiefel manifold $E$ from Table 1 which contains an orthogonal basis for the linear space over $\boldsymbol{k}$ spanned by $x_{1}, \ldots, x_{n}$. Finally, notice that $\pi^{-1}(Z), Z \in S$, consists of only one orbit of $O(1, m-1 ; k)$ if and only if it consists of only closed Lorentz orbits or, equivalently, if and only if $r>m-2$ for $\boldsymbol{k}=\boldsymbol{C}$ and $Z \notin S_{n r-}(\boldsymbol{R})$ for $\boldsymbol{k}=\boldsymbol{R}, r \leqq m-2$, where $r=\operatorname{rank} Z$.

We now discuss the problem mentioned in Introduction.

Remark 7. If $V$ is a differentiable manifold (resp. an analytic set), we denote by $\mathscr{C}^{\infty}(V)$ [resp. $\left.\mathscr{H}(V)\right]$ the linear space of all complex- 
valued differentiable (resp. holomorphic) functions on $V$ endowed with the usual topologies [14]-[16]. Here and throughout this remark "differentiable" means "infinitely differentiable" and the differentiable manifolds may have corners (see [16] for the construction of differentiable structures). Moreover, the analytic structure of $V$ is considered in the sense of Grauert and Remmert [3,15]. If $V$ is a differentiable Lorentz invariant submanifold (resp. an analytic subset) of $M_{m}^{n}(\boldsymbol{k})$, let $\mathscr{C}_{L}^{\infty}(V)$ [resp. $\left.\mathscr{H}_{L}(V)\right]$ denote the subspace of $\mathscr{C}^{\infty}(V)$ for $\boldsymbol{k}=\boldsymbol{R}$ [resp. $H(V)$ for $\boldsymbol{k}=\boldsymbol{C}]$ consisting of all Lorentz invariant functions $\varphi \in \mathscr{C}^{\infty}(V)$ [resp. $\varphi \in \mathscr{H}(V)]$; here $\varphi$ is said to be Lorentz invariant if $\varphi=\varphi_{\Lambda}$ for any $\Lambda \in O(1, m-1 ; \boldsymbol{k})$, where $\varphi_{\Lambda}\left((x)_{n}\right)=\varphi\left(\left(\Lambda x_{1}, \ldots, \Lambda x_{n}\right)\right),(x)_{n} \in M_{m}^{n}(\boldsymbol{k})$.

Theorem 1 and Lemma 1 induce an isomorphisms $i(V, \boldsymbol{R}): \mathscr{C}_{L}^{\infty}(V)$ $\rightarrow \mathscr{C}^{\infty}(\varrho(V))$ [resp. $\left.i(V, \boldsymbol{C}): \mathscr{H}(V) \rightarrow \mathscr{H}(\varrho(V))\right]$ provided $V$ is a Lorentz invariant differentiable submanifold (resp. a Lorentz invariant analytic subset) of the stratum $M \in \tilde{M}_{m}^{n}(\boldsymbol{R})$ [resp. $\left.M \in \tilde{M}_{m}^{n}(\boldsymbol{C})\right]$ and $\varrho(V)$ is canonically identified to a differentiable submanifold (resp. an analytic subset) of $S \times W$ with $M \subset M^{\prime}$ and $M^{\prime}, S$, and $W$ as in Table 3 . In these conditions, the $n$-point Lorentz invariant differentiable (resp. holomorphic) functions can be represented by differentiable (resp. holomorphic) functions of scalar products and elements of Grassmann manifolds. Moreover, this result can be generalized as follows:

If $V$ is a Lorentz invariant differentiable submanifold (resp. a Lorentz invariant analytic subset) of $M_{m}^{n}(\boldsymbol{R})$ [resp. $\left.M_{m}^{n}(\boldsymbol{C})\right]$ such that there exists a nonnegative integer $s$ with

$$
\min _{\substack{(y)_{n} \in V \\ \pi\left((y)_{n}\right)=\pi\left((x)_{n}\right)}} \operatorname{rank}[y]_{n}=\operatorname{rank} \pi\left((x)_{n}\right)+s
$$

for any $(x)_{n} \in V$, then there exists an isomorphism $i(V, \boldsymbol{R})[$ resp. $i(V, \boldsymbol{C})]$ of $C_{L}^{\infty}(V)$ [resp. $\left.\mathscr{H}_{L}(V)\right]$ onto $\mathscr{C}^{\infty}(\varrho(V))$ [resp. $\left.\mathscr{H}(\varrho(V))\right]$ such that its restriction to any stratum $M$ of $\tilde{M}_{m}^{n}(\boldsymbol{R})\left[\operatorname{resp} . \tilde{M}_{m}^{n}(\boldsymbol{C})\right]$ with $M \cap V$ nonvoid is $i(V \cap M, R)$ [resp. $i(V \cap M, C)]$. By $(4.1), \varrho(V)$ can be identified to a differentiable submanifold (resp. an analytic subset) of the union of the manifolds $S \times W$ with $M \cap V$ nonvoid, where $M, S$, and $W$ are given by Table 3. Notice that the case $\boldsymbol{k}=\boldsymbol{C}$ and $s=0$ represents an important theorem of Bargmann, Hall, Wightman [2], and Hepp [3].

The preceding functional formulation of Theorem 1 is an immediate consequence of the stratification of $M_{m}^{n}(\boldsymbol{k})$ and certain extension theorems (see Hepp's method for $\boldsymbol{k}=\boldsymbol{C}$ [3] and the following theorem of Mather for $\boldsymbol{k}=\boldsymbol{R}$ : of $Q$ is a differentiable manifold and $P$ a closed differentiable submanifold of $Q$, then there exists a continuous extension $\eta: \mathscr{C}^{\infty}(P)$ $\rightarrow \mathscr{C}^{\infty}(Q)$ such that $\left.\eta(\varphi)\right|_{P}=\varphi$ for any $\varphi \in \mathscr{C}^{\infty}(P)$ [15], Chapter II, $\S 4$; the Hepp method can be extended for $\boldsymbol{k}=R$ if in Mather's theorem $P$ and $Q$ are appropriate closures of the strata of $M_{m}^{n}(R)$ ). 
We recall that the study of functions invariant under the connected Lorentz group can be reduced to the study of functions invariant under the full Lorentz group using the division theorems for infinitely differentiable and holomorphic functions [17] with respect to the polynomials $\operatorname{det}\left(x_{1}^{\mu}\right)$, where $i=i_{1}, \ldots, i_{m} \in\{1, \ldots, n\} ; \mu=0,1, \ldots, m-1$, and $(x)_{n} \in M_{m}^{n}(k)[1]$.

Let $\mathscr{D}^{\prime}(V)$ denote the topological dual of the Schwartz space $\mathscr{D}(\hat{V})$ of all complex-valued (infinitely) differentiable functions with compact support defined on the differentiable manifold $V[14]$ (for many details of distributions on manifolds see [14], Chapter IX). If $V$ is a differentiable Lorentz invariant submanifold of $M_{m}^{n}(R)$, the space $\mathscr{D}_{L^{+}}^{\prime}(V)$ of even Lorentz invariant distributions [resp. the space $\mathscr{D}_{L-}^{\prime}(V)$ of odd Lorentz invariant distributions] consists of all $f \in \mathscr{D}^{\prime}(V)$ such that $f(\varphi)=f\left(\varphi_{\Lambda}\right)$ [resp. $\left.f(\varphi)=\operatorname{sgn}\left(\Lambda_{0}^{0}\right) f\left(\varphi_{\Lambda}\right)\right]$ for any $\varphi \in \mathscr{D}(V)$ and $\Lambda \in O(1, m-1 ; \boldsymbol{k})$.

Suppose that $V$ is a differentiable Lorentz invariant submanifold of $M_{m}^{n}(\boldsymbol{R})$ which satisfies (4.1) (with $\left.s=0,1\right)$. Denote by $V^{\prime}$ the submanifold of $V$ consisting of all $(x)_{n} \in V$ with $\pi\left((x)_{n}\right) \notin S_{n r-}(\boldsymbol{R}), r=1, \ldots, m-2$. Then there exists an isomorphism of $\mathscr{D}_{L+}^{\prime}(V)$ [resp. $\mathscr{D}_{L_{-}}^{\prime}(V)$ with $V^{\prime}$ nonvoid] onto $\mathscr{D}^{\prime}(\varrho(V))$ [resp. $\left.\mathscr{D}^{\prime}\left(\varrho\left(V^{\prime}\right)\right)\right]$ which carries each $f \in \mathscr{D}_{L^{+}}^{\prime}(V)$ [resp. $\left.g \in \mathscr{D}_{L-}^{\prime}(V)\right]$ to $\hat{f} \in \mathscr{D}^{\prime}(\varrho(V))\left[\right.$ resp. $\left.\hat{g} \in \mathscr{D}^{\prime}\left(\varrho\left(V^{\prime}\right)\right)\right]$ such that

$$
\begin{aligned}
f(\varphi) & =\hat{f}\left(\int_{E} \varphi d \mu(\underline{e})\right), \\
g\left(\varphi \operatorname{sgn}\left(e_{1}^{0}\right)\right) & =\hat{g}\left(\int_{E} \varphi \operatorname{sgn}\left(e_{1}^{0}\right) d \mu(\underline{e})\right),
\end{aligned}
$$

where $\varphi \in \mathscr{D}(V \cap M), \tilde{M} \in M_{m}^{n}(\boldsymbol{R}), E$ is the Stiefel manifold isomorphic to the Lorentz orbits contained in $V \cap M$, and $d \mu(\underline{e})$ is a Lorentz invariant measure on $E$. By (4.3), any $g \in \mathscr{D}_{L_{-}}(V)$ is vanishing on $V \backslash V^{\prime}$. Then the preceding assertion follows immediately from Theorem 1 and certain results of Schwartz (see [14], Chapter IX, $\S 5$; see also [5] for the case $n=1)$.

Acknowledgements. We would like to thank Dr. C. Teleman for many useful suggestions. One of the present authors (El. M.) is grateful to Profs. N. N. Bogoliubov, R. Thom, and A. S. Wightman for stimulating conversations.

\section{References}

1. Weyl, H.: The classical groups, their invariants and representations. Princeton N.J.: Princeton University Press 1939

2. Hall, D., Wightman, A. S.: Math. Fys. Medd. Danske Vid. Selsk. 31, No. 5 (1957)

3. Hepp, K.: Math. Ann. 152, 149 (1963)

4. Minkowski,P., Williams,D.N., Seiler, R.: In: Brittin,W.E., Barut,A.O. (Eds.): Lectures in theoretical physics, Vol. VII A. Boulder, Colorado: University of Colorado Press 1965 
5. Gårding, L., Lions, J.: Nuovo Cimento, Suppl. 14, 9 (1959)

6. Steenrod, N.: The topology of fibre bundles. Princeton Mathematical Series 14. Princeton, N.J.: Princeton University Press 1951

7. Artin, E.: Geometric algebra. New York: Interscience 1957

8. Serre, J.-P.: Lie algebras and Lie groups. Lectures given at Harvard University 1965

9. Loeffel, J. J. : Helv. Phys. Acta 36, 216 (1963)

10. Gantmacher,F.R.: The theory of matrices. Moskow: Nauka 1966 (Russian)

11. Thom, R., Levine, H.: Singularities of differentiable mappings. Bonner mathematische Schriften 6. Bonn: 1959

12. Byers, N., Yang, C. N.: Rev. Mod. Phys. 36, 595 (1964)

13. Gheorghe,A., Ion, D. B., Mihul,El.: To appear

14. Schwartz, L.: Théorie des Distributions. Paris: Hermann 1968

15. Grauert, H., Remmert, R.: Math. Ann. 136, 245 (1958)

16. Mather, J. N.: Structural stability of mappings. Mimeographed notes. Princeton: 1966

17. Mather,J.N.: The division theorems for infinitely differentiable functions and holomorphic functions. Mimeographed notes. Princeton: 1966

Communicated by A. S. Wightman
A. Gheorghe
El. Mihul
Joint Institute for Nuclear Research
Dubna, USSR 